Check for updates

Cite this: RSC Adv., 2017, 7, 47999

\title{
Recent progress in the preparation and application of quantum dots/graphene composite materials
}

\author{
Ling Tong, (D) abc Feng Qiu, *abce Tianjian Zeng, ${ }^{\text {abc }}$ Jia Long, ${ }^{\text {abc }}$ Jie Yang, ${ }^{\text {abc }}$ \\ Rongfei Wang, (D) abc Jin Zhang, ${ }^{\text {bcd }}$ Chong Wang, *abc Tao Sun ${ }^{\star a b c d}$ and Yu Yang*abcd
}

Quantum dots/graphene (QDs/GR) composite materials show a distinct synergistic effect between the QDs and graphene, which has aroused vast attention toward their unique characteristics in the last few decades. The separation of electron-hole pairs generated in QDs are enhanced due to the photogating effects in the composites, and then the electrons or holes are rapidly transferred to the surface of the graphene sheets, resulting in excellent optical gain, making them promising materials for applications in optoelectronic and light harvesting devices. In this review, recent research progress on QDs/GR composites is reviewed and discussed, including preparation methods and applications. Numerous self-assembly synthetic methods such as hydrothermal, solvothermal, atomic layer deposition, ion beam sputtering deposition, and other typical synthetic methods like drop-casting, spin-coating, layer by layer, ultrasonication, polymethylmethacrylate (PMMA) aid transfer and electrochemical methods are summarized and discussed. In addition, the applications of QDs/GR composites such as in photodetectors, lithium ion batteries, solar cells, photocatalysis, electrochemiluminescence (ECL) sensors and supercapacitors are described in detail. Finally, the prospects of QDs/GR composites are discussed.

Received 8th August 2017

Accepted 8th September 2017

DOI: $10.1039 / \mathrm{c} 7 \mathrm{ra0} 8755 \mathrm{a}$

rsc.li/rsc-advances micromechanical cleavage method; ${ }^{15}$ the research on graphene and its various derivatives has become a hot topic globally. Due to its unique properties such as high carrier mobility $(\sim 1 \times$ $10^{3} \mathrm{~cm}^{2} \mathrm{~V}^{-1} \mathrm{~s}^{-1}$ ), high specific surface area and excellent optical transparency, graphene is prospected as an ideal photonic and optoelectronic material, ${ }^{16}$ and is successfully applied in optoelectronic devices ${ }^{17}$ and energy storage devices. ${ }^{18}$ Because of its various advantages, more and more devices will be equipped with graphene or graphene-based composite materials in the future. Nevertheless, QDs and graphene also have their disadvantages. The low carrier mobilities limit the optical gain of QDs materials. Graphene also has drawbacks that cannot be ignored, like its low light absorption, the ultrafast recombination of photo-generated carriers, ease of aggregation and low dispersion. These shortages hinder the use of these two materials in future optoelectronic devices. In recent years, graphenebased nanocomposites combined with nanoparticles have attracted significant attention. Since QDs/GR composite materials were synthesized in 2010, ${ }^{19,20}$ these drawbacks of QDs and graphene have been completely overcome by the synergistic effects of the composite materials. In this hybrid structure, the QDs act as light-absorbing materials to produce electron-hole pairs under the irradiation of light. The carriers can then be rapidly transferred to graphene and produce photocurrent, leading to the efficient separation of electrons and holes and the prolongation of the lifetime of the carriers. The photogenerated carriers in QDs can therefore be effectively transferred to the surface of graphene sheets, which results in high
${ }^{e}$ National Laboratory for Infrared Physics, Shanghai Institute of Technical Physics,

Chinese Academy of Sciences, Shanghai 200083, China

${ }^{a}$ Institute of Optoelectronic Information Materials, School of Materials Science and Engineering, Yunnan University, Kunming 650091, China. E-mail: fengqiu@ynu.edu. cn;.sun@griffith.edu.au

University, Kunming 650091, China

'Yunnan Key Laboratory for Micro/Nano Materials \& Technology, Yunnan University, Kunming 650091, China. E-mail: cwang@ynu.edu.cn

${ }^{d}$ School of Energy, Yunnan University, Kunming 650091, China. E-mail: yuyang@ynu. edu.cn 
optical gain, excellent optical response and good photo catalytical activities (shown in Scheme 1(a)-(c)). The charge transfer mechanism is the basic principle in the QDs/GR hybrid system. Based on the synergetic and cooperative effects of the hybrid, some new phenomena and physical properties can be inspired. Owing to their excellent performances, graphene inorganic nanocomposites have become a new class of electrocatalysts. ${ }^{21}$ QDs/GR composite materials can therefore provide a novel strategy for low-cost and high performances for potential applications in optoelectronic and light harvesting devices. ${ }^{19,22,23}$ Recently, these materials have been applied in photodetectors, ${ }^{24-27}$ lithium ion batteries, ${ }^{28,29}$ solar cells, ${ }^{30,31}$ photocatalysis, ${ }^{32,33}$ ECL sensors ${ }^{34}$ and other devices.

Aktürk et al. using first-principles calculations, demonstrated that $\mathrm{Si}$ and Ge atoms were bound at the bridge sites on graphene with binding energies of $0.54 \mathrm{eV}$ and $0.49 \mathrm{eV}$ at a coverage of $\theta=1 / 32 .{ }^{35}$ The variable energy of $\mathrm{Si}$ and Ge atoms was most likely diffused along the T B H T sites, and the energy barrier was too low to prevent the clustering of adatoms; however, the binding energy in the $\mathrm{H}$ site was higher than the energies of the $\mathrm{T}$ and $\mathrm{B}$ sites. The $\mathrm{H}$ site adatom proved to be the tightest to combine with $\mathrm{C}$ atoms in graphene, and $\mathrm{Si}$ atoms showed higher binding energies than Ge atoms, regardless of being in the $\mathrm{H}$ site or $\mathrm{T}$ site, as shown in Fig. 1(a). The energy bands and density of states in the coverage of $\theta=1 / 32$ are shown in Fig. 1(b). The doping of Si and Ge atoms changed the graphene band structure and narrowed the band-gap, which benefited the carrier migration. Besides, the unbalanced spins demonstrated the charge transfer from adatoms to graphene.

The photogating effect was firstly discovered and named by Hou et al. using the analysis of microelectronic and photonic structures (AMPS) computer model in 1992. ${ }^{36}$ Their computer simulation results showed that this effect could be ascribed to the trapped charge and low field region in front of amorphous Si-based $p-i-n$ structures under bias light illumination. They also demonstrated that the photogating effect was responsible for quantum efficiencies greater than unity. The photogating effect was further analyzed in forward biased a-Si:H Schottky barrier structures, ${ }^{37}$ reverse biased a-Si:H based $\mathrm{p}-\mathrm{i}-\mathrm{n}$ junctions $^{38}$ and microcrystalline silicon $(\mu \mathrm{c}-\mathrm{Si}: \mathrm{H})$ based $\mathrm{n}-\mathrm{i}-\mathrm{p}$
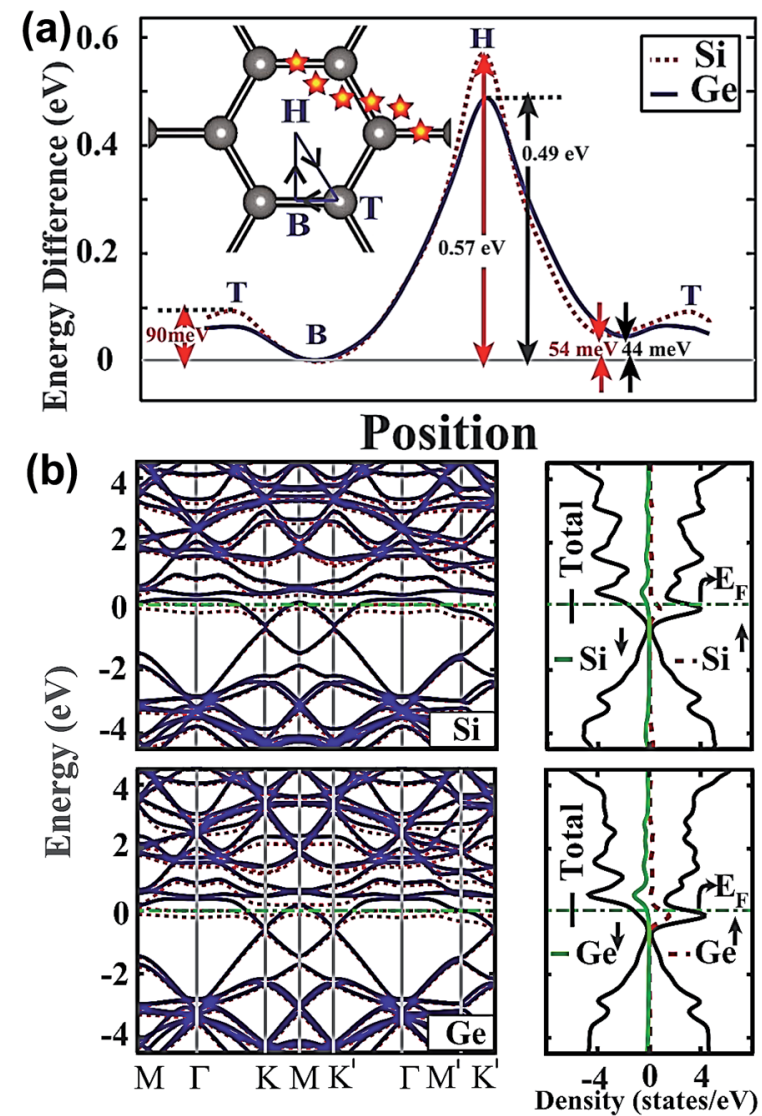

Fig. 1 (a) Binding energy of Si and Ge adatoms in different sites, and their migration path. The adsorption sites were divided and named, top $(\mathrm{T})$, bridge $(\mathrm{B})$ and hollow $(\mathrm{H})$, as shown in the map inserted in (a). (b) Energy band structure and total density of states of $\mathrm{Si}$ and Ge atoms adsorbed to graphene in the $(4 \times 4)$ supercell. Reprinted with permission from ref. 35. Copyright 2010 American Institute of Physics.

devices. ${ }^{39}$ It was found that the photogating effect was derived from band tail states in the conduction and valence bands of semiconductors, which were caused by structural defects and disordered interfaces. The band tails could also trap the photogenerated electrons/holes, prolong the lifetimes of carriers
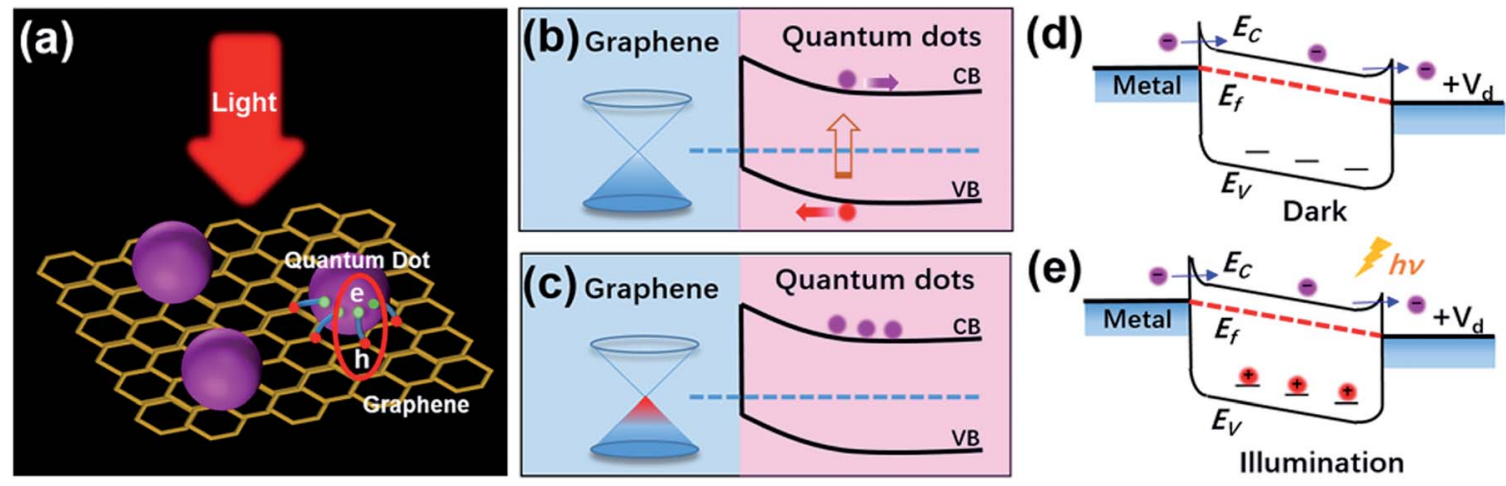

(e)

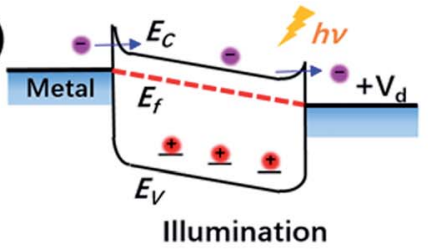

Scheme 1 (a) Schematic illustration of the QDs/GR composite and light-generated electron-hole pairs. Energy level diagrams of the QDs/GR interface showing (b) electron-hole pair generation in QDs under illumination and (c) graphene doping induced by trapped carriers. Schematic of the photogating effect (d) under external bias in the dark and (e) under illumination. 
and form built-in fields, as shown in Scheme 1(d) and (e). ${ }^{40}$ In QDs/GR composites, photoconductive gain and responsivity could be effectively enhanced by the photogating effect, having huge potential for the application in optoelectronic devices.

In 2015, Khan et al. reviewed the synthesis, properties and applications of graphene based metal and metal oxide nanocomposites in detail. ${ }^{\mathbf{4 1}}$ The preparation methods were broadly classified as top-down and bottom-up methods. The graphenebased inorganic nanocomposites show great potential in the wide-range applications of the electronics, electrochemical and electrical fields. In 2016, Shi et al. reported the synthesis and applications of 3D graphene-based composites. ${ }^{42}$ They mainly discussed the synthesis methods and applications in energy storage and conversion as electrode materials. At the same time, Pham et al. reviewed the graphene-semiconductor quantum dot hybrids in the application of photovoltaics and photodetectors. ${ }^{43}$ Particularly, the in situ and ex situ synthesis methods and charge transfer in hybrid systems were presented.

In this review, recent progress of QDs/GR composite materials, especially the synthesis method and its applications, are briefly reviewed. The challenges of the technique and its future development are discussed. Due to the unique synergistic effects between QDs and graphene, the QDs/GR composite materials have demonstrated excellent properties in optoelectronic, electrochemical and energy storage devices in recent years, and will be among the most promising hybrid materials in the near future.

\section{Preparation methods}

The conventional methods for synthesizing QDs are mainly chemical vapor deposition (CVD), sol-gel, molecular beam epitaxy (MBE), liquid phase epitaxy (LPE), etc. Graphene was initially produced by micromechanical exfoliation, but liquidphase exfoliation, carbon segregation and CVD have been exploited in the last few decades; the QDs/GR composite materials can also be easily prepared by these methods. Selfassembly methods can transform these systems from the disordered to ordered state by their internal interactions, which are considered to be much more useful routes, and are described in detail in the following sections.

Table 1 List of synthetic methods for the preparation of different QDs/GR composite materials and their applications

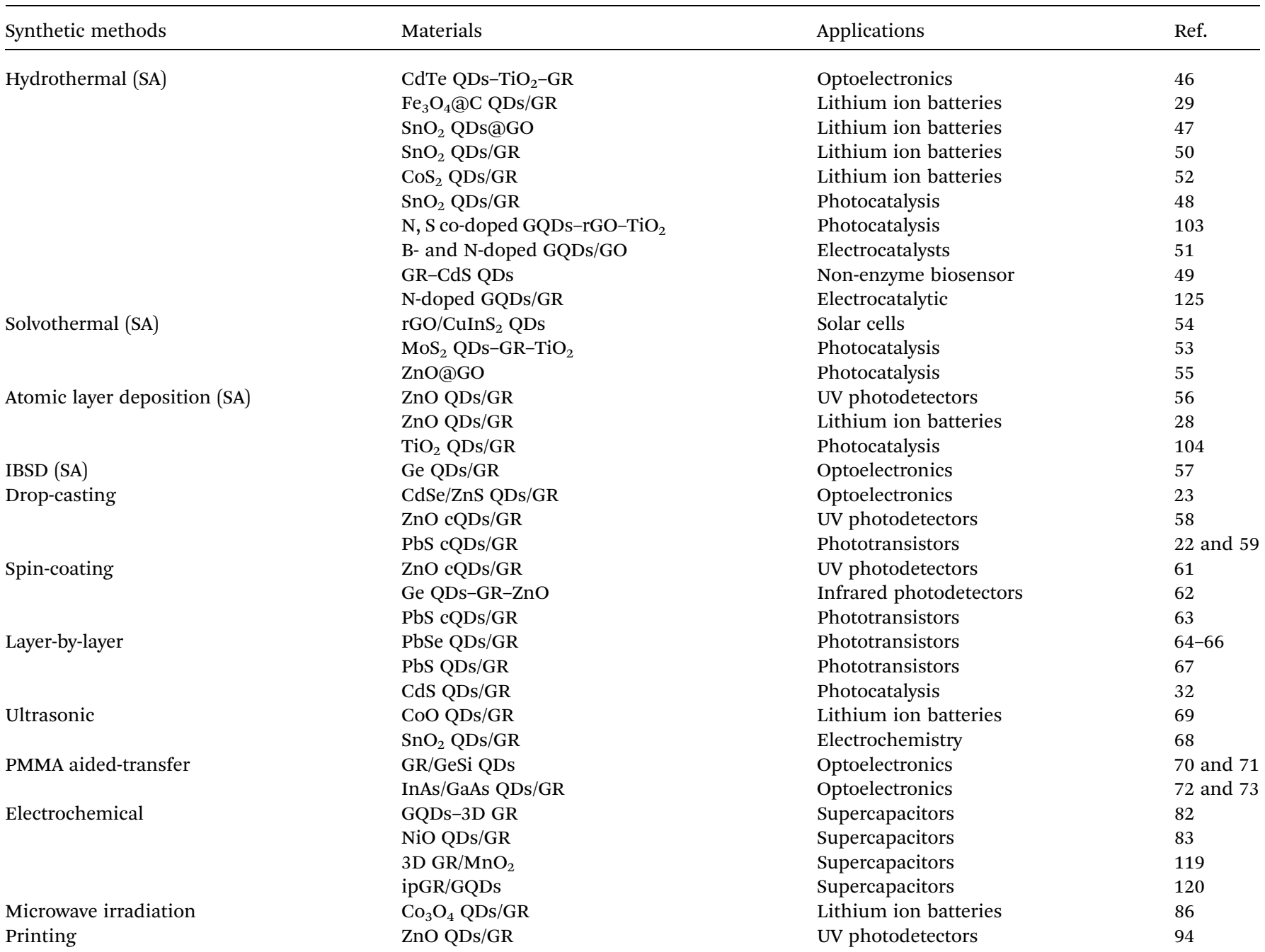


Due to the high surface area and ultrahigh conductivity, graphene is often employed as a fine conductive layer in hybrid materials, in which electrons or holes can be effectively transferred, and leads to good photoelectric performances. However, pure graphene film has limited functional groups, and restrains the possibility for chemical modification. Thus, graphene oxide (GO) and reduced graphene oxide (rGO) have come to be ideal materials because they possess lots of oxygen-containing functional groups and defects, such as hydroxyl, carboxyl, carbonyl and epoxy groups, and numerous nano-scale materials can be associated with them via covalent or noncovalent bonds. GO and rGO are convenient for use in solvent processes to promote the syntheses of hybrid structure and aid the transfer of electrons or holes. The colloidal quantum dots (cQDs) can also possess lots of chemical bonds and ligands on its surface, which act as outstanding connecting mediums and carrier channels.

So far, numerous synthetic methods have been employed to prepare different QDs/GR composite materials; chemical methods are mainly used due to their low cost. These methods are summarized in Table 1.

\subsection{Self-assembly methods (SA)}

Self-assembly methods have been widely employed for QDs synthesis, although the self-assembly concept was first used in bioscience. Self-assembly involves processes that can promote the disordered components turning into ordered patterns by their internal interactions. It has three remarkable features: (I) the components may react directly toward a high ordered state; (II) the weak interactions such as, van der Waals $\pi-\pi$, hydrogen bonds, etc., play important roles in determining their physical properties; (III) the building blocks are broad, and not only include atoms and molecules, but also contain nano- and mesoscopic structures.

To date, numerous trials have been carried out to develop new materials for adopting self-assembly methods. The synthetic routes are designed to expand applications and cut costs; some typical self-assembly methods such as hydrothermal, solvothermal, atomic layer deposition, ion beam sputtering deposition, are introduced and summarized.

2.1.1 Hydrothermal method. The hydrothermal method is a useful and simple one for materials synthesis. In this process, the insoluble species are dissolved or recrystallized in a highpressure atmosphere. There are several advantages to using this method: (I) it can prepare some special materials at a relatively low heating temperature, (II) the chemical reaction rate in the hydrothermal process is faster. For example, the hydrothermal heating temperature is only $80-240{ }^{\circ} \mathrm{C}$ for preparing $\mathrm{BaCO}_{3}$ powders, compared to the traditional industrial route that requires a calcination temperature of $1000-1200{ }^{\circ} \mathrm{C} .{ }^{44}$ Due to its low energy consumption and simple experimental requirements, $\mathrm{Fe}_{3} \mathrm{O}_{4}$ QDs/GR composite, ${ }^{45} \mathrm{Fe}_{3} \mathrm{O}_{4} @ \mathrm{C}$ QDs/GR composite, ${ }^{29}$ CdTe QDs- $\mathrm{TiO}_{2}-\mathrm{GR}$ composite, ${ }^{46} \mathrm{SnO}_{2}$ QDs@GO composite, ${ }^{47} \mathrm{SnO}_{2}$ QDs/GR composite, ${ }^{48}$ etc., have been synthesized by the hydrothermal method in recent years.

Zhao et al. ${ }^{47}$ recently employed GO as a template for growing oxidized $\mathrm{SnO}_{2}$ QDs. $\mathrm{Sn}^{2+}$ ions were oxidized by the functional groups of GO, and GO was applied as the template for promoting selective heterogeneous nucleation; the produced $\mathrm{SnO}_{2}$ nanoparticles were nucleated and grown on the surface of GO. The $\mathrm{SnO}_{2}$ QDs@GO composite was formed during the hydrothermal process, as illustrated in Fig. 2(a). Fig. 2(b) shows the transmission electron microscopy (TEM) image of $\mathrm{SnO}_{2}$ QDs@GO with good dispersion and high mass loading. As shown in Fig. 2(c) and (d), $\mathrm{SnO}_{2}$ QDs preferred to grow along the [001] direction, with (110) surfaces as oxygen bridging sites on the $\mathrm{SnO}_{2}$ (110) plane. After assembling into LIBs, the huge volume expansion/contraction of lithium-ion batteries during the processes of discharging/charging was effectively released. This composite was also beneficial for fast lithium intercalation/de-intercalation, due to the existence of graphene.

In 2014, Zhang et al. reported that the GR-CdS QD hybrids could be used for non-enzyme biosensors via the one-step hydrothermal method. ${ }^{49}$ GO was first prepared from graphite by a modified Hummer's method, then mixed with $70 \mathrm{~mL}$ water to obtain the GO dispersions by the ultrasonication process. When the weight ratio of $\mathrm{GO}$ and $\mathrm{Cd}\left(\mathrm{CH}_{3} \mathrm{COO}\right)_{2} \cdot 2 \mathrm{H}_{2} \mathrm{O}$ was $0.5 \%$ (G0.5-CdS), the graphene-CdS QDs composite with a good distribution and high coverage rate was prepared via non-covalent bonding. In this process, thiourea and $\mathrm{Cd}\left(\mathrm{CH}_{3} \mathrm{COO}\right)_{2} \cdot 2 \mathrm{H}_{2} \mathrm{O}$ were employed as the sulphur source and cadmium source, respectively.

Polyacrylic acid (PAA), for example, possesses lots of carboxylic acid groups, which urges the link between QDs and GO and promotes the coverage rate and uniformity. It also benefits from the negative charges, such as $\mathrm{COO}^{-}$and $\mathrm{OH}^{-}$, because they can attach to CdS QDs to make them more stable.

As shown in Fig. 3(a), CdS QDs were excited, and yielded photo-generated electrons and holes under illumination, then

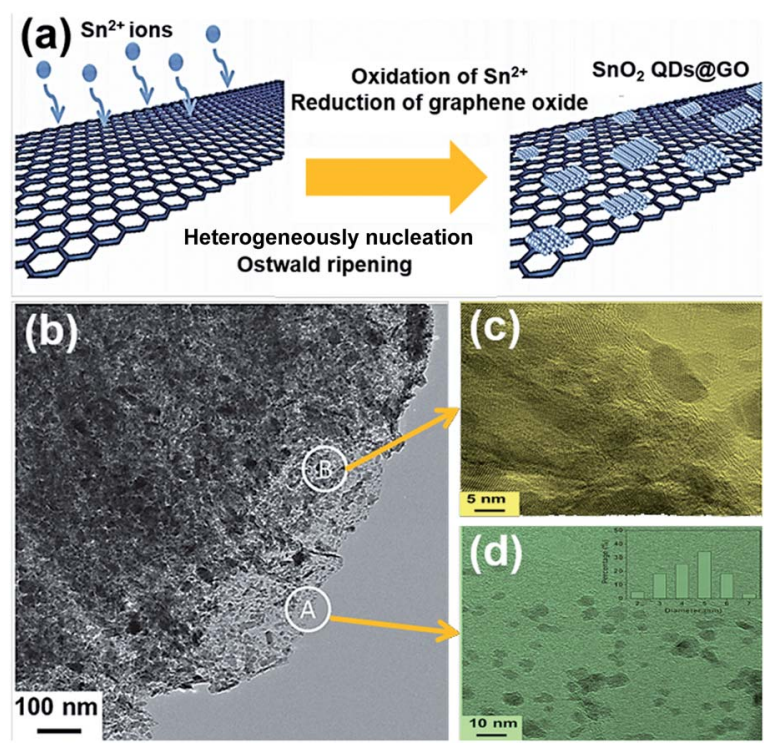

Fig. 2 (a) Schematic illustration for the fabrication of $\mathrm{SnO}_{2}$ QDs@GO. (b) TEM image of $\mathrm{SnO}_{2}$ QDs@GO. (c), (d) High-resolution transmission electron microscope (HRTEM) of section $B$ and A in image (b), respectively. The inset in image (d) is a size distribution image. Reprinted with permission from ref. 47. Copyright 2015 Wiley-VCH. 
the electrons were rapidly transferred to graphene film from the conduction band (CB) of CdS QDs. Graphene acted as the electron capture and transport matrix to avoid electron-hole recombination, resulting in prolonging the electron lifetime and high light sensitivity. In this process, the $\mathrm{OH}^{-}$was oxidized to $\mathrm{OH}$ by photo-generated holes, so that glucose was oxidized to gluconolactone by $\mathrm{OH}^{*}$ and was further detected through different photocurrent densities (Fig. 3(d)). The lattice fringes of cubic CdS particles in the G0.5-CdS hybrid can be seen clearly in Fig. 3(b), while CdS QDs stacked randomly together when prepared without GO. It is believed that the graphene could promote crystal growth and in turn prevent itself aggregation with the CdS QDs loading. The G0.5-CdS/ITO electrode has long lifetime and high photocurrent density with glucose, as shown in Fig. 3(c) and (d).

Liu et al. prepared the CdTe $\mathrm{QDs}-\mathrm{TiO}_{2}$-graphene hybrid via a general hydrothermal method. ${ }^{46}$ The CdTe QDs-TiO $_{2}$-graphene hybrid was fabricated by the in situ growth of CdTe QDs on the $\mathrm{rGO}-\mathrm{TiO}_{2}$ composite surface. The fluorescence quenching of the produced hybrid indicates that the electrons transfer from CdTe QDs to $\mathrm{TiO}_{2}$ and graphene. Additionally, Ye et al. fabricated the $\mathrm{SnO}_{2}$ QDs/GR composite with visible light catalytic activity via a one-step, low cost and environment-friendly hydrothermal process ${ }^{48}$ that also avoided the agglomeration of QDs. Zhao et al. prepared a carbon-coated $\mathrm{Fe}_{3} \mathrm{O}_{4}$ QDs/GR composite by using a hydrothermal and calcining route. ${ }^{29}$ In this process, the carbon shell can prevent the aggregation and buffer the volume expansion, which improves lithium-storage performance.

Some novel technologies combined with hydrothermal methods have been employed in recent years. Huang et al. successfully fabricated the $\mathrm{SnO}_{2}$ QDs/GR composite via the
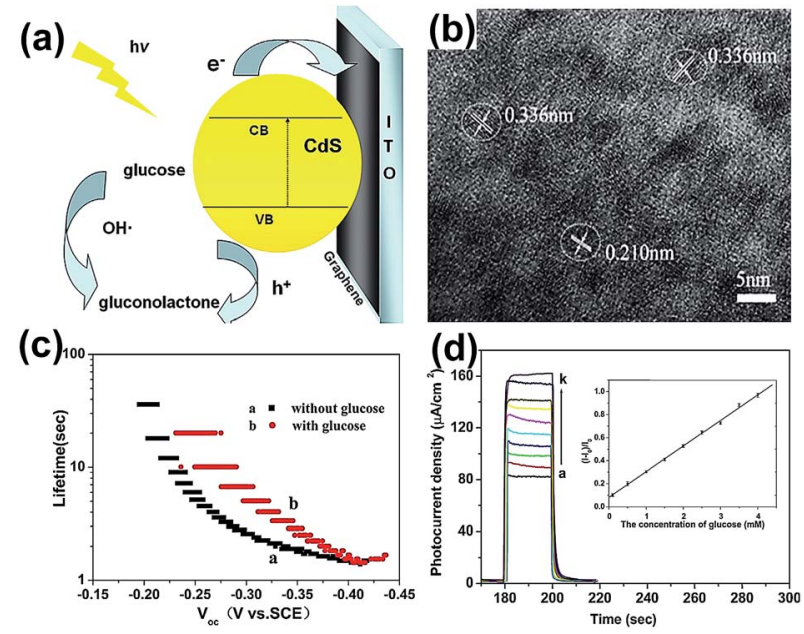

Fig. 3 (a) Schematic diagram for the photoelectrochemical process of oxidation of glucose and electron transfer at the G0.5-CdS/ITO electrode. (b) HRTEM image of G0.5-CdS. (c) Electron lifetime versus open-circuit potential for the electrode without and with glucose. (d) Photocurrent density of the electrode in $\mathrm{NaOH}$ in the presence of different concentrations of glucose (concentration increased from bottom to top). Inset: linear calibration curve. Reprinted with permission from ref. 49. Copyright 2014 Elsevier Ltd. electron-beam irradiation (EBI) technique combined with the hydrothermal method in 2015..$^{50}$ The EBI was mainly used for irradiating the graphene nanosheets to increase the interlayer spacing; it also promoted exfoliation and removed the partial oxygen-containing functional groups. The SEM figures show that the $\mathrm{SnO}_{2}$ QDs were loaded on both sides of the graphene sheets with uniform dispersion. The high reversibility and specific capacity as well as good cycle response of this composite material promote its use in the energy storage field. Besides, the B- and N-doped graphene quantum dots/GO (GQDs/GO) composite was prepared via a self-assembled hydrothermal treatment in a sealed Teflon-lined autoclave, following the co-doping of $\mathrm{B}$ - and $\mathrm{N}$ - by high-temperature annealing. ${ }^{51}$ This hybrid could be applied as an excellent oxygen reduction electrocatalyst. He et al. synthesized $\mathrm{CoS}_{2}$ nanoparticles wrapped by $\mathrm{CoS}_{2}$ QDs/GR via an L-cysteineassisted hydrothermal reaction. ${ }^{52}$ The $\mathrm{CoS}_{2}$ QDs/GR composite provided more active sites for the intercalation of lithium ions. The graphene in the composite demonstrated high transport abilities of electrons and lithium ions, and could also effectively suppress the volume expansion of $\mathrm{CoS}_{2}$ nanoparticles. Under their synergistic effect, this material exhibits excellent performances for the application of lithium-ion batteries.

2.1.2 Solvothermal method. The solvothermal method uses organics as the solvent instead of water. Since water-sensitive compounds cannot be synthesized via the hydrothermal method, organic solvents are used to replace water, which is an advisable route for preparing the uniformly dispersed QDs on the surface of graphene by controlling its nucleation.

In 2015, Gao et al. synthesized the $\mathrm{MoS}_{2}$ QDs-GR-TiO composite via the one-pot solvothermal method. ${ }^{53}$ Thiocarbamide was applied as the precursor, then mixed with $\mathrm{Na}_{2} \mathrm{MoO}_{4} \cdot 2 \mathrm{H}_{2} \mathrm{O}$ and $\mathrm{P} 25$ powder. The formation mechanism of $\mathrm{MoS}_{2}$ QDs was ascribed to the interactions between the functional groups of GO and Mo precursors as shown in Fig. 4(a). The sizes of $\mathrm{MoS}_{2}$ QDs are 2 to $3 \mathrm{~nm}$ and the lattice fringes from

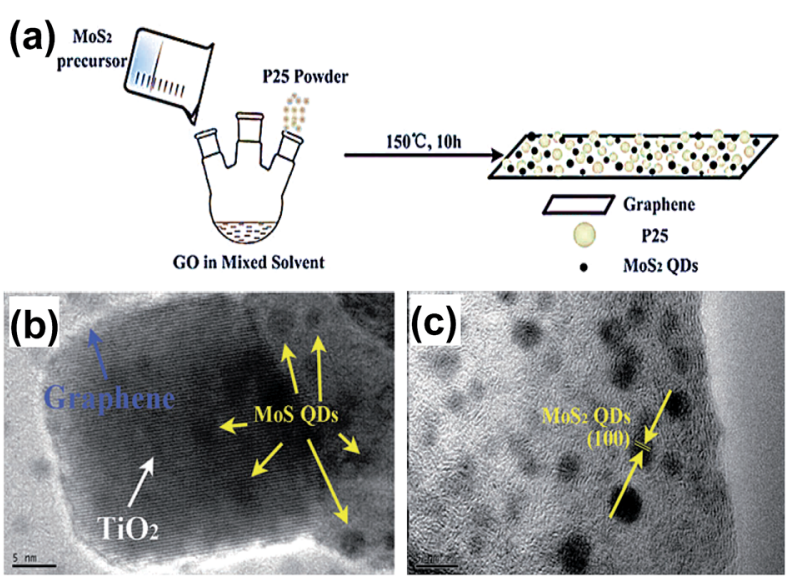

Fig. 4 (a) Schematic demonstration of the fabrication of the $\mathrm{MoS}_{2}$ QDs-graphene- $\mathrm{TiO}_{2}$ composite. (b) HRTEM image of the $\mathrm{MoS}_{2}$ QDsgraphene- $\mathrm{TiO}_{2}$ composite. (c) HRTEM image of the $\mathrm{MoS}_{2}$ QDs-graphene hybrid. Reprinted with permission from ref. 53. Copyright 2015 Royal Society of Chemistry. 
the (100) planes of crystal $\mathrm{MoS}_{2}$ QDs can be observed. The $\mathrm{MoS}_{2}$ QDs-GR hybrid was introduced as a co-catalyst to overcome the low charge separation rate and large band gap caused by $\mathrm{TiO}_{2}$. It also enhanced the visible-light absorption, charge separation and photocatalysis ability, and significantly improved its photocatalysis properties.

Meng et al. synthesized the $\mathrm{rGO} / \mathrm{CuInS}_{2}$ QD hybrid using thiourea as the $\mathrm{S}$ doping source and $\mathrm{CS}\left(\mathrm{NH}_{2}\right)_{2}$ as the precursor by the same approach. ${ }^{54}$ The GO films were reduced to rGO during the formation of $\mathrm{CuInS}_{2}$ QDs, and the adsorbed $\mathrm{Cu}^{+}$was used for the nucleation of $\mathrm{CuInS}_{2}$ QDs. They deemed that the $\mathrm{Cu}^{2+}$ was mainly adsorbed at the epoxy/hydroxyl groups on GO at room temperature. The $\mathrm{rGO} / \mathrm{CuInS}_{2}$ QD hybrid was a good electron acceptor in polymer-based solar cells. The efficiency of assembled solar cells was $1.5 \%$ higher, compared to the rGO sheet counterpart devices. Atchudan et al. fabricated the ZnO@GO composite via the solvothermal method. ${ }^{55}$ The GO sheet base was synthesized by utilizing the Hummers method, and the added $\mathrm{ZnO}$ nanoparticles were prepared by thermal oxidation. The $\mathrm{ZnO}$ nanoparticles were then impregnated into the GO surface for ZnO@GO composite preparation via the solvothermal process. The $\mathrm{ZnO}$ nanoparticle sizes were about 10-20 nm. This composite can be applied as a photocatalyst for organic dye degradation.

The one-pot solvothermal method is relatively simple and easy to control. The chemical reaction in this process could be carried out in a hermetic system, which could effectively prevent the volatilization of toxic substances and avoid air, for air sensitive precursors.

2.1.3 Atomic layer deposition method. Atomic layer deposition (ALD) is a useful technique for a single atomic layer film by self-limiting reactions. The surface reaction of ALD results from self-limiting properties, which are fundamental to ALD technology. Shao et al. ${ }^{56}$ synthesized the ZnO QDs/GR composite by the ALD method. The typical chemical reactions were as follows:

$$
\begin{gathered}
\mathrm{ZnOH}^{*}+\mathrm{Zn}\left(\mathrm{CH}_{2} \mathrm{CH}_{3}\right)_{2} \rightarrow \mathrm{ZnOZn}\left(\mathrm{CH}_{2} \mathrm{CH}_{3}\right)^{*}+\mathrm{CH}_{3} \mathrm{CH}_{3} \\
\mathrm{Zn}\left(\mathrm{CH}_{2} \mathrm{CH}_{3}\right)^{*}+\mathrm{H}_{2} \mathrm{O} \rightarrow \mathrm{ZnOH}^{*}+\mathrm{CH}_{3} \mathrm{CH}_{3}
\end{gathered}
$$

in which the asterisks indicate the surface species. The $\mathrm{ZnO}$ nanoparticles were expected to grow on the defects of the graphene surface. They found that the ZnO QDs were deposited on both the tops and sides of the graphene sheets, and the size was about $9 \mathrm{~nm}$. The ZnO QDs/GR composite was a sandwich structure obtained via spin coating in conductive polymers, and was used as a photodetector. Under the synergistic effects caused by the highly photoactive ZnO QDs and high carrier mobility of graphene, the produced photodetector achieved high photoresponsivity, owing to the produced active QDs/GR composite material.

Sun et al. prepared the ZnO QDs/GR composite by the same approach for enhancing the capacity of lithium storage. ${ }^{28}$ The growth rate of $\mathrm{ZnO}$ was about $2 \AA$ per cycle, thus, it was easy to control the $\mathrm{ZnO}$ particle sizes during the ALD cycles. The fabricated $\mathrm{ZnO}$ QD sizes were 2 to $7 \mathrm{~nm}$ through the ALD process. The ALD reaction process and images for characterization are shown in Fig. 5. The sizes of $\mathrm{ZnO}$ nanoparticles were about $2 \mathrm{~nm}$ and $7 \mathrm{~nm}$ after 15 and 50 ALD cycles, respectively. The increase in the sizes was nearly a linear function of the cycling numbers. Previously, the $\mathrm{ZnO}$ nanoparticles were hard to nucleate directly on graphene, due to the unreactive properties of graphene. The $\mathrm{ZnO}$ nanoparticles were expected to nucleate at the edge planes and defects because of the probable lower energy on those sites.

2.1.4 Ion beam sputtering deposition method. Recently, our group employed a novel strategy to grow Ge QDs directly on a graphene $/ \mathrm{SiO}_{2} / \mathrm{Si}$ substrate by the ion beam sputtering deposition (IBSD) technique, which provided a new route for the low cost and extensive industrialization of Ge QDs/GR composites. ${ }^{57}$ By this physical self-assembly method, Ge atoms were deposited on graphene sheets without the aid of organic or inorganic ligands, which is unlike the chemical method. The entire process was carried out under vacuum. The graphene/ $/ \mathrm{SiO}_{2} / \mathrm{Si}$ substrates were deposited with Ge atoms for 60, 180, and 300 seconds at room temperature, respectively. The fourth sample was then grown at $500{ }^{\circ} \mathrm{C}$ for $180 \mathrm{~s}$ as contrast. The atomic force microscopy (AFM) morphology images are shown in Fig. 6(a), (b) and (c), respectively. The process of evolution indicates that Ge atoms firstly nucleate on the surface of graphene, and gather into Ge islands by self-assembly. The peaks shown in the XPS results are $29.2 \mathrm{eV}$ for $\mathrm{Ge}-\mathrm{Ge}$ and $30.7 \mathrm{eV}$ corresponds to $\mathrm{Ge}-\mathrm{C}$ bonds, which significantly confirm the interfacial interaction between Ge QDs and graphene. The photoluminescence (PL) spectra exhibited different luminescence peak positions from Si-based Ge QDs as shown in Fig. 6(e); it was ascribed to no $\mathrm{Si}-\mathrm{Ge}$ intermixing and the effect of surface states in Ge QDs. Owing to the advantages of low-cost and high efficiency to prepare high quality QDs, the IBSD method is considered to be a powerful tool to prepare QDs/GR composites.

In general, the self-assembly methods that have been utilized to synthesize QDs/GR composites are efficient. It is
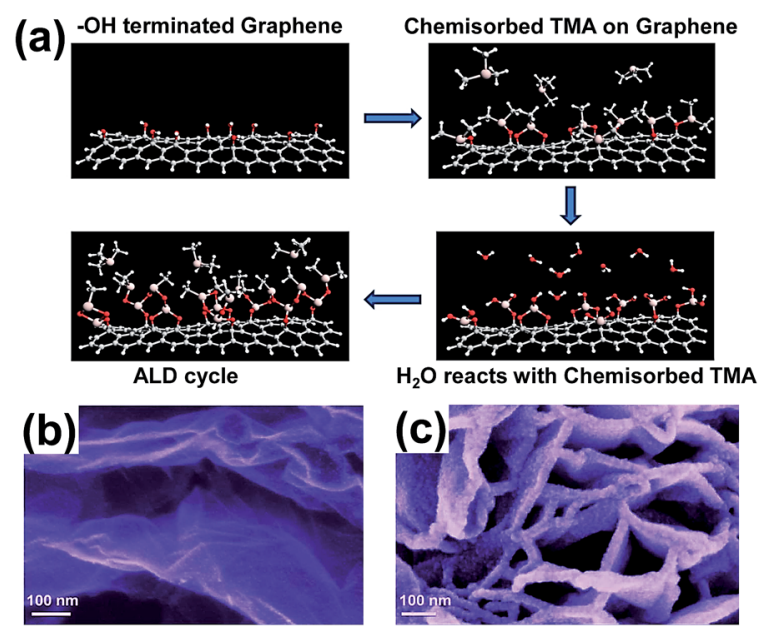

Fig. 5 (a) Schematic diagram of ALD reaction. SEM images of the $\mathrm{ZnO}$ QDs/GR composite at 15 ALD cycles (b) and 50 ALD cycles (c). Reprinted with permission from ref. 28. Copyright 2014 Royal Society of Chemistry. 

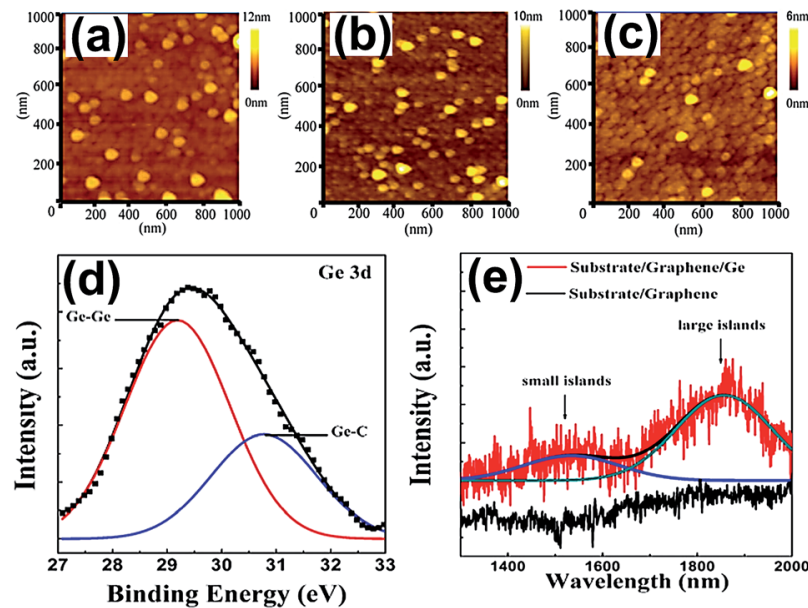

Fig. 6 AFM images of the Ge QDs/GR composite at different sputtering times at room temperature: $60 \mathrm{~s}$ (a), $180 \mathrm{~s}$ (b) and $300 \mathrm{~s}$ (c). XPS image (d) and PL spectra (e) of the Ge QDs/GR composite grown at $500^{\circ} \mathrm{C}$. Reprinted with permission from ref. 57. Copyright 2016 IOP Publication Ltd.

critical to choose suitable building blocks and preparation processes. The chemical bonds that link QDs with graphene and provide the channels for the transfer of separated electrons or holes lead to the enhanced electrochemical and photoelectric properties, which satisfy the growing market requirements from industry, customers and the military.

Except for the SA methods, drop-casting, spin-coating, layer by layer, ultrasonic, PMMA aided-transfer and other typical methods may also be employed to synthesize QDs/GR composite materials. In recent years, tremendous efforts have been made for QDs/GR composite fabrication and property improvement via these methods. With the aid of external conditions, composites can be prepared via directional manipulation and fast reaction.

\subsection{Drop-casting method}

The drop-casting method is a frequently implemented strategy to prepare films. The procedure is as follows: several solutions are dropped onto a substrate without rotation, and are then heated for hours or annealed for several minutes to remove water. In recent years, some groups have successfully prepared QDs/GR composites such as the CdSe/ZnS QDs/GR composite, ${ }^{23}$ the colloidal ZnO QDs/GR composite, ${ }^{58}$ the PbS QDs/GR composite ${ }^{22,59}$ and giant nanocrystal QDs/GR composite,$^{60}$ using this method.

In 2015, Gromova et al. prepared colloidal core/shell CdSe/ ZnS QDs by hot-injection synthesis, and multi-layer graphene flakes were prepared by ultrasound processing of graphite. ${ }^{23}$ The solution containing CdSe/ZnS QDs material was deposited on the multi-layer graphene sheets to fabricate the CdSe/ZnS QDs/GR composite via the drop-casting method. The extraordinary mechanical properties are as a result of the multi-layer graphene structure, which causes the composite to be easily processed in solution; the hybrid structure enhances the photoelectrical response.
Son et al. fabricated ultraviolet (UV) photodetectors using the ZnO cQDs/GR hybrid. ${ }^{58}$ Firstly, the GR/PET virtual substrate was fabricated using PMMA to transfer graphene onto the PET substrate, then the solution containing ZnO cQDs was deposited onto the surface of graphene to fabricate the $\mathrm{ZnO}$ cQDs/GR composite. The synthesis process is shown in Fig. 7(a). This composite possessed a high optical transmittance $(\sim 80 \%)$ and good flexibility (Fig. 7(b)). The HRTEM image suggests that the colloidal ZnO QDs are tightly attached to the surface of the graphene sheet, as shown in Fig. 7(c).

Turyanska et al. employed ligands to combine colloidal $\mathrm{PbS}$ QDs with graphene for fabricating the PbS cQDs/GR hybrid using the drop-casting method..$^{59}$ Various ligands such as polyethylene glycol, thioglycerol (TGL) and 2,3-dimercapto-1propanol (DTG) with different lengths were explored. Polyethylene glycol $\mathrm{H}-\left(\mathrm{O}-\mathrm{CH}_{2}-\mathrm{CH}_{2}\right)_{n}-\mathrm{OH}$ with $n=2000$ and $n=500$ was denoted $\mathrm{QD}_{\mathrm{P} 2000}$ and $\mathrm{QD}_{\mathrm{P} 500}$; the corresponding lengths were 10 and $5 \mathrm{~nm}$, respectively. The $\mathrm{QD}_{\mathrm{TGL}}$ represents the thioglycerol capped QDs with $0.5 \mathrm{~nm}$ length. As shown in Fig. 8, the graphene Fermi energy increased by $+50 \mathrm{meV}$, but was still below the QD LUMO state after depositing $\mathrm{QD}_{\mathrm{P} 2000}$ and $\mathrm{QD}_{\mathrm{P} 500}$; the conduction was p-type at $V_{g}=0$ after current versus $V_{g}$ characteristics and calculations. The photo-generated electrons in QDs were transferred to graphene's LUMO energy level (Fig. 8(b)). When $\mathrm{QD}_{\mathrm{TGL}}$ was deposited, the Fermi energy shifted by $+300 \mathrm{meV}$, and the conduction became n-type at $V_{\mathrm{g}}=0$; the photo-generated holes in QDs preferred to be transferred to the graphene sheet (Fig. 8(c)). The characteristics in Fig. 8(d) indicate ultra-high responsivity $\left(\sim 10^{9} \mathrm{~A} \mathrm{~W}^{-1}\right)$, which is two orders

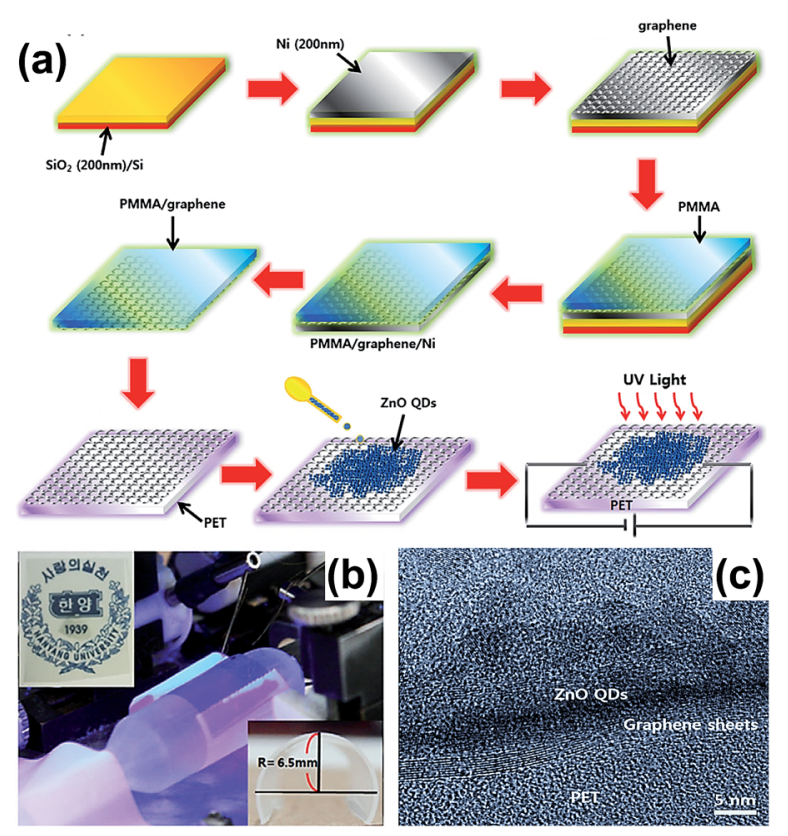

Fig. 7 (a) Illustration of the preparation of the $\mathrm{ZnO}$ QDs/GR composite. (b) Images of the equipment and device; the inset on the bottom right shows a flexible UV photodetector. (c) HRTEM image of the $\mathrm{ZnO}$ cQDs/GR composite on a polyethylene terephthalate (PET) substrate. Reprinted with permission from ref. 58. Copyright 2015 Elsevier Ltd. 

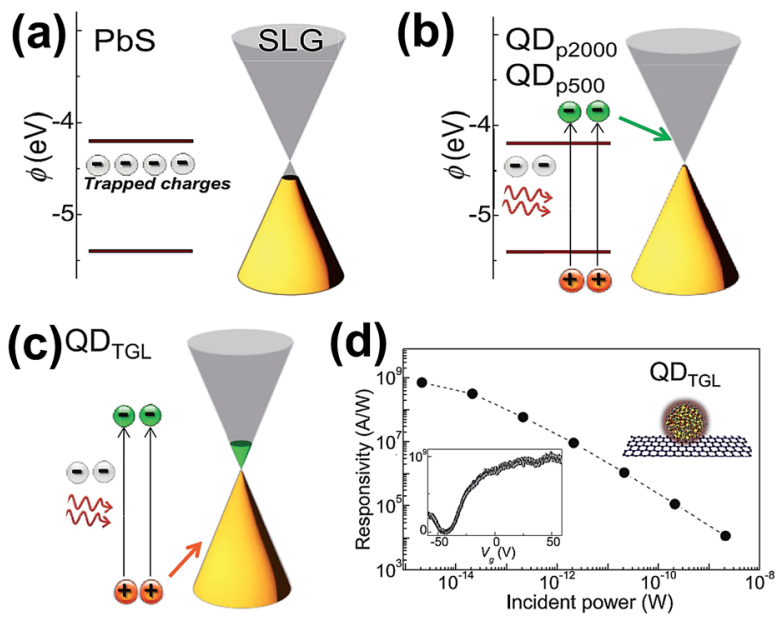

Fig. 8 Schematic illustration of the energy states (a) before depositing QDs on graphene, (b) after depositing $\mathrm{QD}_{\mathrm{P} 2000}$ and $\mathrm{QD}_{\mathrm{P} 500}$ on graphene and (c) after depositing QD TGL on graphene. (d) Photoresponsivity as a function of the incident light power of the $\mathrm{QD}_{\mathrm{TGL}} / \mathrm{GR}$ transistor device. The bottom left inset shows photoresponsivity as a function of gate voltage $\left(V_{\mathrm{g}}\right)$. Reprinted with an Open Access from ref. 59. Published by WILEY-VCH Verlag Gmbh \& Co. KGaA, 2015.

higher than in other previous reports. This extraordinary performance can be ascribed to the charge transfer from QDs and the accumulation of charge. The responsivity of the transistor can also be enhanced by choosing ligands of suitable length and preserving their integrity.

\subsection{Spin-coating method}

The spin-coating method is another similarly useful path to dropcasting. The difference between them is that this process requires a rotating substrate for spin-coating. The thickness of the film can be easily controlled by rotating speed and running time.

Ultraviolet (UV) photodetectors based on a colloidal $\mathrm{ZnO}$ QDs/GR hybrid have been fabricated via the wet spin-coating process. ${ }^{61}$ The colloidal ZnO QDs were synthesized and dropped onto the graphene flakes, then the composite was annealed for few minutes for residual solvent elimination. The distribution of ZnO QDs was uniform on the graphene sheets. The final manufactured UV photodetector showed a high ratio of the photocurrent to the dark current $\left(\sim 1.1 \times 10^{4}\right)$.

Liu et al. fabricated an infrared photodetector on a flexible substrate by the analogic method. ${ }^{62}$ The Ge QDs-GR-ZnO heterostructure resulted in high responsivity $\left(\sim 9.7 \mathrm{~A} \mathrm{~W}^{-1}\right)$ in a shortwave infrared field $(1400 \mathrm{~nm})$ and fast response speed $(\sim 40 \mu$ sise time and $90 \mu \mathrm{s}$ recovery time). Konstantatos et al. ${ }^{63}$ fabricated phototransistors based on a PbS cQDs/GR hybrid; the specific detectivity was up to $7 \times 10^{13}$ Jones, benefitting from the photogating effect of the trapped electrons in PbS QDs and forming a built-in field at the QDs/GR interface, the responsivity was $10^{7} \mathrm{~A} \mathrm{~W}^{-1}$.

\subsection{Layer by layer method}

The layer by layer (LbL) method is an effective strategy for obtaining the multilayer QDs/GR hybrid structure, and many hybrids have been employed for field effect phototransistors (FEpTs) in recent years. For realizing ultrahigh photoresponsivity, the QDs acted as the light absorption layer, and graphene was the charge transfer layer. Zhang et al. designed a PbSe QDs/GR hybrid; ${ }^{64}$ a wet chemical method was applied for $\mathrm{PbSe}$ QDs preparation and the graphene was prepared by a CVD technique and subsequently transferred to the substrate. One drop of PbSe QD solution was deposited on a rotating substrate, and then three drops of ethanedithiol (EDT) solution were placed on the same substrate for ligand exchange. Four layers of PbSe QDs were deposited on graphene. The absorption and emission peaks were $1616 \mathrm{~nm}$ and $1645 \mathrm{~nm}$, respectively. The electrons and holes of the produced FEpTs achieved high mobilities and the light responsivity was $1 \mathrm{~A} \mathrm{~W}^{-1}$.

In 2015, two types of multi-heterojunction FEpTs were prepared based on a PbSe QDs/GR hybrid with different layer arrangements via the same approach, denoted QD-bottom and G-bottom. ${ }^{65}$ Fig. 9(a)-(d) shows the schematic diagrams and cross-sectional SEM images of QD-bottom FEpTs and G-bottom FEpTs. The thickness of the $\mathrm{SiO}_{2}$ layer was $300 \mathrm{~nm}$, and the thickness of the QD-bottom FEpTs (109 nm) was close to G-bottom FEpTs (103 nm), which demonstrated that the uniformity of the QDs is better than for graphene in the first layer with thickness of $103 \mathrm{~nm}$. There were slight point shifts for QD-bottom FEpTs and large negative shifts for G-bottom FEpTs,
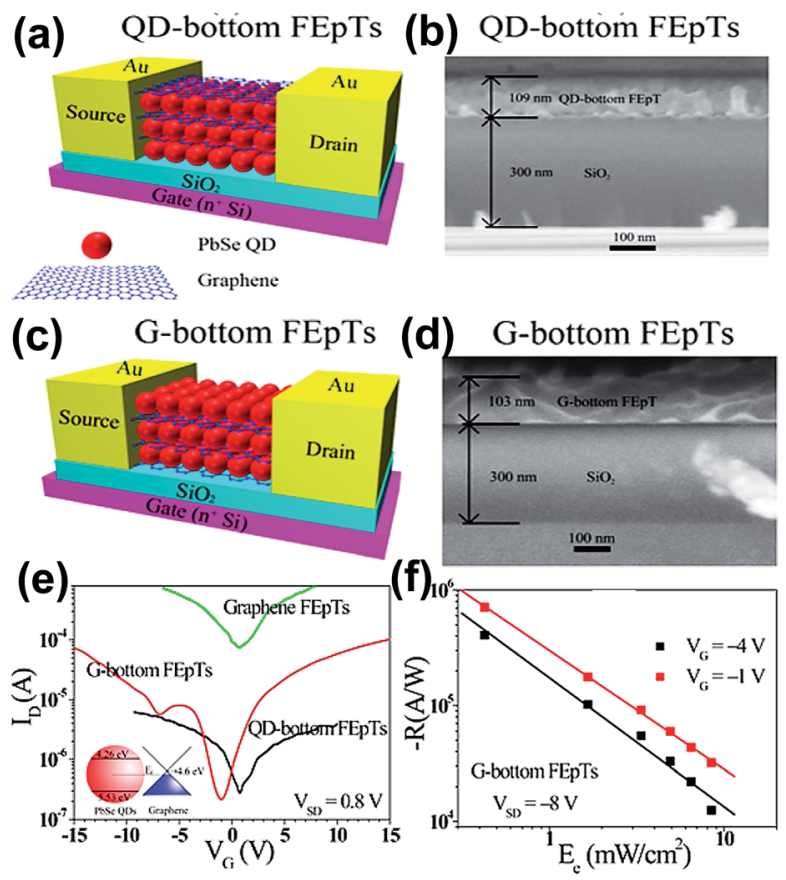

Fig. 9 Schematic diagram of QD at the bottom layer FEpTs (a) and the corresponding cross-sectional SEM images of the multi-heterojunction channel (b). Schematic diagram of graphene at the bottom layer of FEpTs (c) and the corresponding cross-sectional SEM images of the multi-heterojunction channel (d). (e) Transfer characteristics of graphene FEpTs, G-bottom FEpTs and QD-bottom FEpTs without light illumination. (f) Responsivity as a function of illumination of the G-bottom FEpTs. Reprinted with permission from ref. 65. Copyright 2015 American Chemical Society. 
as shown in the transfer characteristic curves in Fig. 9(e). As is well known, the shift degree is related to the QD surface defects, the degree of energy level matching and the barriers induced by ligands. The changes in the transfer curve demonstrate that the carriers were transferred from QDs to graphene following the Dirac point shift. Fig. 9(f) shows that the responsivity was up to $10^{6} \mathrm{~A} \mathrm{~W}^{-1}$; the G-bottom layer demonstrated multi-saturation behaviour, due to the back-gate effect.

The same group also reported another two types of multiheterojunction FEpTs based on the same hybrid material to compare their photoresponse properties. ${ }^{66}$ They demonstrated that the layered heterojunction FEpTs were better candidates for near infrared phototransistors than bulk heterojunction FEpTs. Meanwhile, the wide spectral response FEpTs based on $\mathrm{PbS}$ QDs/GR hybrids were also investigated by the above group via the layer by layer method. ${ }^{67}$ Because of the multiple-exciton generation of PbS QDs and its synergistic effects with graphene, the assembled FEpTs achieved a wide spectral response (300$1400 \mathrm{~nm})$ and high responsivity $\left(2 \times 10^{5} \mathrm{~A} \mathrm{~W}^{-1}\right.$ at $10 \mathrm{mV}$ bias voltage).

The layer by layer strategy can also be employed for selfassembly. In 2004, Liu et al. successfully fabricated a CdS QDs/GR hybrid to study its photoelectrochemical and photocatalytic properties. ${ }^{32}$ In the fabrication processes, the graphene flakes were obtained by reduction of graphite oxide, then the clean substrate was dipped into CdS QDs mixed with thioglycolic acid (TGA) solution and graphene nanosheetspoly(allylamine hydrochloride) (PAH) solution. These procedures were repeated to fabricate the multi-layered films. The photocatalytic and photoelectrochemical activities of the new CdS QDs/GR multilayered were remarkably enhanced.

\subsection{Ultrasonic method}

The ultrasonic method is another effective technique for QDs/ GR composite material preparation. Chen et al. fabricated a $\mathrm{SnO}_{2}$ QDs/GR composite via a facile one-step ultrasonic approach by using $\mathrm{SnCl}_{2} \cdot 2 \mathrm{H}_{2} \mathrm{O}$ as the precursor. ${ }^{68}$ The sizes of the $\mathrm{SnO}_{2}$ QDs were 4-6 nm, and the synthesized QDs were uniformly dispersed on both sides of the graphene. The schematic of the mechanism of formation is shown in Fig. 10(a). Firstly, the $\mathrm{Sn}^{2+}$ ions of the $\mathrm{SnCl}_{2}$ solution combined with GO through electrostatic attraction to the carbonyl and carboxyl groups, then ultrasonic vibration was used to increase the interlayer space to make $\mathrm{Sn}^{2+}$ migrate into the enlarged layer, which resulted in prohibiting the GO stacks and enhancing the $\mathrm{Sn}^{2+}$ loading number. Finally, the $\mathrm{SnO}_{2}$ QDs/GR composite was obtained through a calcination treatment. Fig. 10(b) shows the uniformly distributed $\mathrm{SnO}_{2}$ QDs on the graphene sheet with good crystallinity. In Fig. 10(c), the lattice fringes can be clearly observed, indicating that the $\mathrm{SnO}_{2}$ was well-crystallized with the rutile phase (110) face. The typical XRD patterns are shown in Fig. 10(d); the bare graphene sheets (\#1) showed a strong (002) diffraction peak, and the similarity of the diffraction peaks of the $\mathrm{SnO}_{2}$ QDs/GR hybrid (\#2) and $\mathrm{SnO}_{2}$ nanoparticles (\#3) indicates that the $\mathrm{SnO}_{2}$ QDs were well-crystallized in the hybrid. However, the (002) diffraction peak is not sharp in the XRD of

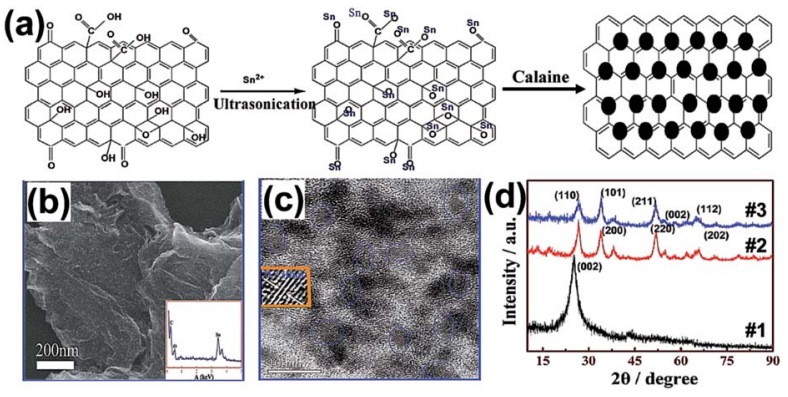

Fig. 10 (a) Schematic diagram of the formation of a $\mathrm{SnO}_{2} \mathrm{QDs} / \mathrm{GR}$ hybrid. (b) Field emission-secondary electron microscopy (FE-SEM) image of the $\mathrm{SnO}_{2}$ QDs/GR hybrid on the $\mathrm{Si}(100)$ substrate. (c) HRTEM image of the $\mathrm{SnO}_{2}$ QDs/GR hybrid. (d) XRD patterns of bare graphene sheets (\#1), $\mathrm{SnO}_{2}$ QDs/GR hybrid (\#2) and $\mathrm{SnO}_{2}$ nanoparticles (\#3). Reprinted with permission from ref. 68. Copyright 2013 American Chemical Society.

the $\mathrm{SnO}_{2}$ QDs/GR hybrid (\#2), which might be caused by the graphene sheets' regular lamellar structure, which was broken and turned into exfoliated graphene in the synthetic processes. These phenomena also indicate that both sides loaded with $\mathrm{SnO}_{2}$ QDs by this novel method can prevent graphene restacking during the reduction process and the graphene also prevents agglomeration of $\mathrm{SnO}_{2}$ QDs during the calcination. The ultrasonication mainly provides the driving force to enlarge the interlayer spacing of graphene, and supplies more effective loading sites for $\mathrm{SnO}_{2}$ QDs on the graphene surface. Additionally, the ultrasonication could also prevent graphene stacking.

Sonication can be used to provide energy for decomposition in the hybrid process. In 2012, Peng et al. employed $\mathrm{Co}_{4}(\mathrm{CO})_{12}$ as the cobalt precursor and synthesized a CoO QDs/GR composite by the ultrasonic method. ${ }^{69}$ The mechanism of the material formation can be divided into two steps: (I) Co was produced by the decomposed $\mathrm{Co}_{4}(\mathrm{CO})_{12}$ precursor, and deposited on the surface of the graphene sheet with the assistance of sonication. (II) Co was oxidized in situ into CoO. The successful fabrication is ascribed to the strong interaction between electron-rich Co atoms and graphene's electrophilic carbon atoms. The sizes of $\mathrm{CoO}$ QDs were 3 to $8 \mathrm{~nm}$, and the QDs were well-dispersed on the graphene surface. The obtained composite inhibits the aggregation of graphene and the volume changes of $\mathrm{MO}_{x}$ during the cycling test of lithium-ion batteries. As anode material, this composite demonstrates a highly reversible lithium storage capacity and outstanding cycling stability.

\subsection{Polymethylmethacrylate (PMMA) aided-transfer method}

Polymethylmethacrylate (PMMA) is a widely used medium for graphene transfer. The procedures can be divided into three steps as follows: (I) PMMA/GR/substrate is constructed into the sandwich-structure, (II) etching solution or organic solvent is removed from the substrate, (III) PMMA is dissolved in acetone solution or organic solvent.

In 2015, Chen et al. successfully fabricated the GR/GeSi QDs composite by using a PMMA aided-transfer method. $^{70}$ 
Ten-layered GeSi QDs were prepared on Si (001) substrates by the MBE technique, and the graphene sheet was grown by the CVD technique. The monolayer graphene sheet was transferred onto the GeSi QDs surface with the aid of PMMA. The manufactured device structure is shown in Fig. 11(a) and the uniform size distribution of GeSi QDs can be observed in the AFM image. The average size and height of the surface QDs was about 100 and $7 \mathrm{~nm}$, respectively. The schematic energy band diagram of this composite is shown in Fig. 11(b). The potential distribution could promote the separation of photo-generated electron-hole pairs, and the added electric field could reduce the graphene Fermi level, thus the electrons can be transferred from QDs to graphene more easily. The decrease in PL Intensity from 0 to $-40 \mathrm{~V}$ also demonstrated the enhancement of electron transfer, as shown in Fig. 11(c). It was found that the luminescence properties of the composite can be adjusted by laser energy and graphene plasmon with the external vertical field. Similarly, they fabricated the GR/GeSi QD hybrid structures via the same path in $2014 .^{71}$ In particular, a 10-layer GeSi QDs sample was divided into two equal parts, one of them was covered by graphene, and the other part was uncovered. The surface-plasmonpolariton (SPP) enhanced absorption mechanism was proposed to illustrate the enhancement of the photoluminescence (PL) intensity of the graphene/GeSi QD hybrid.

This method was also used to prepare the InAs/GaAs QDs/GR composite. ${ }^{72,73}$ Ajlani et al. synthesized the graphene-capped InAs/GaAs QDs hybrid by this method. InAs QDs were grown by the MBE technique on a GaAs (001) substrate, and multilayer graphene flakes were grown by the CVD technique. The multilayer graphene flakes were transferred onto QDs with the aid of PMMA, and uncapped QDs were designed for comparison. The enhancement of PL was observed, which indicated electron transfer from graphene to QDs. On the other hand, the presence of the cap layer modified the appearance of the QDs, and prevented the surface oxidation; it also acted as the active layer in a laser device.

(a)

(a)

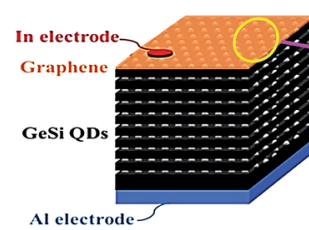

(b)

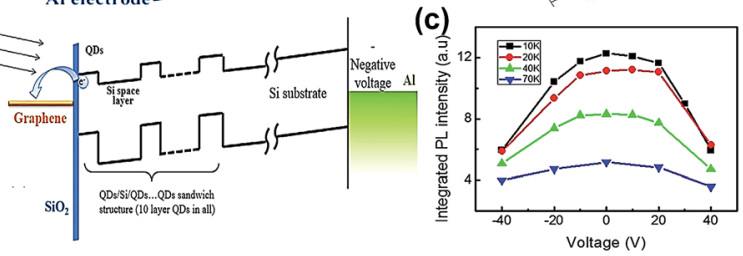

Fig. 11 (a) Schematic illustration of the structure of the ten-layer GeSi QDs/GR composite device and the corresponding AFM image of the top layer GeSi QD sample. (b) Schematic diagrams of energy band alignment under negative voltage. (c) PL spectra of the hybrid structure under -40 to $40 \mathrm{~V}$ voltage at different temperatures. Reprinted with an Open Access from ref. 70. Copyright 2015 Springer Nature.

\subsection{Electrochemical method}

Electrochemical synthesis is an effective strategy for synthesizing nano-scale materials and functionalized composites. Compared to other synthetic methods, the electrochemical method possesses many superiorities such as mild reaction conditions, environmental friendliness, rapidness, cost effectiveness, and simple operation.

In recent years, the electrochemical method has been widely employed in synthesizing QDs and nanoparticles-decorated graphene. For example, GQDs, ${ }^{74} \mathrm{~N}$-doped GQDs, ${ }^{75}$ and $\mathrm{N}$ doped carbon $\mathrm{QDs}^{76}$ have been successfully prepared by the electrochemical method for cellular imaging. They bring numerous advantages of high quantum yield, low biotoxicity and large scale synthesis. In addition, the electrochemically synthesized GQDs ${ }^{77}$ and P-doped GQDs ${ }^{78}$ showed high activity for glucose detection and free radical-scavenging. The $\mathrm{rGO} / \mathrm{ZnO}$ microspheres-Au nanoparticles ${ }^{79}$ and $\mathrm{Au}$ nanoparticles decorated as flower-like graphene ${ }^{\mathbf{8 0}}$ also exhibited high sensitivity for hydrazine and nitrite, respectively.

The QDs/GR composites have also been synthesized via the electrochemical method by Kim et al. in 2010. They prepared homogenous and ordered CdSe QD arrays on the graphene basal plane via electrochemical synthesis. ${ }^{81}$ They used $8 \mathrm{~nm}$ sized hexagonal symmetry vertical structure (SKU-1) as a nanoporous mask to form uniform QDs on the graphene surface. The SKU-1-coated graphene was applied as the working electrode, then CdSe was deposited on it under a constant potential of $-0.7 \mathrm{~V}$ at $50{ }^{\circ} \mathrm{C}$, after which, the SKU-1 template was removed. The nanoporous mask provided resistance to prevent the diffusion of electrolytes. Moreover, the partial deposition on edge sites could be effectively suppressed.

In 2014, Chen et al. also synthesized the GQDs-3D graphene composite by the electrochemical method. ${ }^{82}$ They used carbon rod as the working electrode to form GQDs by cyclic voltammetry (CV). Then, in a three-electrode system, the 3D graphene was used as the working electrode, and the GQDs solution were electrodeposited on the surface of the 3D graphene under a bias at $+2 \mathrm{~V}$ for 5-10 hours in a three-electrode system. They used this composite as an electrode to assemble supercapacitors without binding agent or conduction additive. This supercapacitor demonstrated an excellent electrochemical capacitance (268 $\mathrm{F} \mathrm{g}^{-1}$ ) with $90 \%$ enhancement, compared to that of pure 3D graphene electrodes $\left(136 \mathrm{~F} \mathrm{~g}^{-1}\right)$.

In 2015, Jing et al. prepared a NiO QDs/GR composite through a one-step alternating voltage electrochemical method by using a graphite rod and Ni flakes as the working electrode. ${ }^{83}$ Under alternating voltage, both graphite rod and Ni flakes were exfoliated to form graphene and NiO QDs, respectively; the oxygen functional groups on the graphene surface act as a bridge to link graphene and $\mathrm{NiO}$ through $\mathrm{Ni}-\mathrm{O}-\mathrm{C}$ bands in the electrochemical process. These bands also contributed to enhancing the composite's electrical conductivity, which presented a high specific capacitance of the fabricated supercapacitor $\left(1181.1 \mathrm{~F} \mathrm{~g}^{-1}\right.$ at the current density of $\left.2.1 \mathrm{~A} \mathrm{~g}^{-1}\right)$. Thus, the electrochemical method is a simple, environmentally 
friendly, and controllable technique for QDs/GR composite preparation.

\subsection{Other novel methods}

Other novel methods have also been employed to prepare QDs/ GR composites, such as chemical bath deposition, ${ }^{20,84}$ successive ionic liquid adsorption and reaction ${ }^{85}$ microwave irradiation, ${ }^{86}$ one-step thermal-treatment ${ }^{34}$ and one-step wet chemical methods. ${ }^{87}$ Zhou et al. synthesized a $\mathrm{Co}_{3} \mathrm{O}_{4}$ QDs/GR composite successfully via the microwave irradiation method. ${ }^{86}$ Firstly, the graphene mixture was treated with ultrasound for half an hour, then $\mathrm{Co}\left(\mathrm{CH}_{3} \mathrm{COO}\right)_{2} \cdot 4 \mathrm{H}_{2} \mathrm{O}$ and $25 \% \mathrm{NH}_{3} \cdot \mathrm{H}_{2} \mathrm{O}$ were added to the mixed solution. After sonication, the suspension was transferred into a microwave oven for heating and irradiation. Finally, the mixture was rinsed and dried to obtain the $\mathrm{Co}_{3} \mathrm{O}_{4}$ QDs/GR composite. This composite demonstrated a significantly enhanced cycling performance $\left(1785 \mathrm{~mA} \mathrm{~h} \mathrm{~g}^{-1}\right.$ at $0.1 \mathrm{C}$ after 90 cycles) and high rate capability ( $485 \mathrm{~mA} \mathrm{~h} \mathrm{~g}^{-1}$ at $\left.5 \mathrm{C}\right)$ in the LIBs tests.

In summary, various QDs/GR composites can be prepared by different methods. The hydrothermal and solvothermal methods are limited for materials used in photoelectric devices because it is hard to control the size of QDs, even if the prepared hybrid is uniformly dispersed. Although atomic layer deposition can accurately control the membrane thickness by adjusting the cycling numbers, the short lifetime of the vacuum pump still limits its industrialized production. IBSD is low-cost compared to MBE, but good crystallinity and high density QDs on graphene are not reported. Drop-casting and spin-coating are common approaches for membrane preparation since it could easily control the film thickness, but the solvent significantly impacts the film quality. Besides, electrostatic forces and hydrogen bonds are required to provide the driving force in the layer by layer self-assembly method, and different driving forces will influence the membrane morphology and structure. Ultrasound can provide extra driving forces for the hybrid processes, but the degree of driving forces is hard to control. PMMA aidedtransfer is an established method for graphene transfer, but the graphene quality is destroyed to a certain extent in the transfer processes.

Generally, in choosing a proper method, one should consider the functions and applications of the materials, and the preparation cost. So far, almost all the synthetic techniques are chemical methods, and the physical methods for the direct growth of QDs composites on graphene are rarely investigated; even the ordered and size-tunable QDs have already been prepared on various substrates by IBSD and MBE methods. The physical methods have broad prospects in QDs/GR composite preparation, and it is necessary to further investigate these synthesis techniques in the future.

\section{Applications}

Benefiting from the developments and modifications of the QDs/GR composites synthesis technique, the QDs/GR composite based devices generate several excellent photoelectric and high capacity performances in photoelectric conversion and energy-storage devices. For example, with the remarkable high carrier mobility, this material brings ultrahigh responsivity to photodetectors, for applications in the lithium ion batteries and solar cells, and the QDs/GR composites have excellent storage capacity and conversion efficiency. The QDs decorated on graphene sheets in the application of photocatalysis can effectively increase the visible-light absorbance and charge separation, which remarkably increases the photocatalysis ability. For the QDs/GR composites based electrochemiluminescence sensors, the sensitivity is high for biomedical detections and chlorinated phenols, which play a significant role in medical and environmental techniques.

\subsection{Photodetectors}

Photodetectors are the devices that turn the optical signals into electric signals. As is well known, semiconductor QDs are superior photoelectric conversion materials, and graphene is an excellent electron conduction material. QDs combined with graphene could achieve high performance photodetectors. According to the response wavelength, photodetectors can be divided into infrared light, visible light and ultraviolet photodetectors. A photodetector is an important type of sensor in optical-fiber communication systems, and can be widely applied in the military, industrial production and daily equipment. With the development of science and technology, the performances of photodetectors including their responsivity and sensitivity require improvement.

In 2014, Pham et al. observed the quenching of the thiolfunctionalized $\mathrm{rGO}$ ( $\mathrm{TrGO}$ ) electron paramagnetic resonance (EPR) signal in CdSe QDs-TrGO composites, which was ascribed to electronic coupling and direct chemical attachment. ${ }^{88}$ In 2016, they demonstrated that the surface defects of ZnO nanoparticles could be effectively healed by sulfur and thiol groups of TrGO via the investigation of EPR. ${ }^{89}$ Besides, the thiol groups demonstrated a higher healing ability than oxygencontaining functionalities. The electrons were proved to transfer from ZnO nanoparticles to the lattice of TrGO, and their spins coupled with localized electron spins might take responsibility for the quenching of the TrGO EPR signal. Moreover, the enhanced green defect emission was proven to be derived from the charge transfer from graphene to the defect states of ZnO nanoparticles. These studies have significant influence on the research and application of photodetectors.

The colloidal ZnO QDs/GR composite, synthesised by the wet spin-coating technique, was employed in ultraviolet (UV) photodetectors. ${ }^{61}$ The ratio of photocurrent to dark current $\left(I_{\text {light }} / I_{\text {dark }}\right)$ was approximately $1.1 \times 10^{4}$ with $365 \mathrm{~nm}$ wavelength illumination. The rise and decay times were $2 \mathrm{~s}$ and $1 \mathrm{~s}$, respectively. This photodetector achieved high responsivity and quick response for UV light. The photoresponse mechanisms were ascribed to the photogeneration-recombination of the photo-generated electron-hole pairs and the adsorption-photodesorption on the ZnO QD surface. The ALD-grown ZnO QDs/ GR composite processed high sensitivity for UV light and the maximum photoresponsivity was measured to be $247 \mathrm{~A} \mathrm{~W}^{-1}$ at 
$325 \mathrm{~nm}^{.56}$ It is widely known that the high active surface enhances the materials' photoresponsivity.

The PbS QDs coated graphene composites for use in the near infrared (NIR) photoconductor have the responsivity of $10^{7} \mathrm{~A}$ $\mathrm{W}^{-1}$, which was much higher than the responsivity of the visible-light detector based on the $\mathrm{PbS}$ thin film-graphene composite. ${ }^{90}$ The high absorption coefficient of QDs and the excellent conductivity of graphene cooperate with the Fermi level modulated increase in the responsivity of the devices. Moreover, the infrared photodetector based on QDs decorated $\mathrm{rGO} / \mathrm{ZnO}$ heterostructure shows a high responsivity at $9.7 \mathrm{~A}$ $\mathrm{W}^{-1}{ }^{34}$ Zeng et al. ${ }^{91}$ fabricated the graphene/germanium heterojunction and used it in an infrared photodetector; the $I_{\text {light }} /$ $I_{\text {dark }}$ ratio was $2 \times 10^{4}$ at zero bias voltage, which is about two times higher than that synthesized by Son et al. ${ }^{61}$ The properties, including the reproducibility, spectral selectivity and stability, were also improved.

In 2016, Haider et al. reported an ultrahigh responsivity photodetector by using graphene and GQDs on the $\mathrm{Pb}\left(\mathrm{Zr}_{0.2} \mathrm{Ti}_{0.8}\right)$ $\mathrm{O}_{3}$ (PZT) substrate via the hydrothermal and spin coating technique. ${ }^{92}$ The electric field $\left(E_{\mathrm{P}}\right)$ pointed downward PZT-based device was denoted as DPZT, and the $E_{\mathrm{P}}$ pointed upward PZTbased device was denoted as UPZT. The prepared composite was annealed to improve the junction between graphene and GQDs. The average size of GQDs was 5-7 $\mathrm{nm}$. As shown in Fig. 12(a) and (b), the sensitivity of DPZT reached to $4.06 \times 10^{9} \mathrm{~A}$ $\mathrm{W}^{-1}$, which is two orders higher than the reported relevant devices $\left(\sim 10^{7} \mathrm{~A} \mathrm{~W}^{-1}\right)$, and the gain achieved $10^{10}$ under UV light illumination. The realization of ultrahigh sensitivity was mainly ascribed to the intrinsic electric field from PZT and the built-in electric field from the QDs/GR hybrid, which greatly enhanced the separation of photogenerated electron-hole pairs and forced the holes transferred from QDs to the graphene channel, respectively. This technique can be applied in quantum information technology, optical communication, optoelectronic circuits, etc. Fig. 12(c) shows the energy band diagrams; there are many defects that exist on the PZT surface, and the electrons

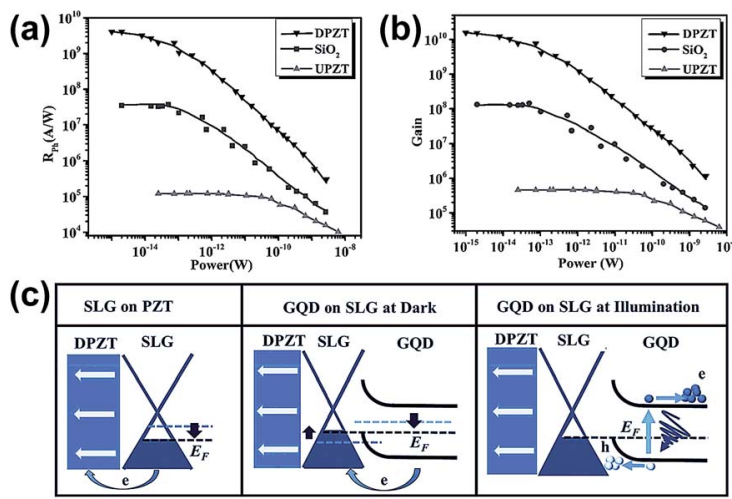

Fig. 12 Photoresponsivity $\left(R_{\mathrm{Ph}}\right)$ (a) and photocurrent gain (b) as a function of applied power for different substrates. (c) Schematic energy band diagrams for different devices and conditions. Reprinted with permission from ref. 92. Copyright 2015 WILEY-VCH Verlag $\mathrm{GmbH} \&$ Co. KGaA. in single layer graphene (SLG) are transferred to the interface defect states of PZT after depositing graphene on it, which reduces the graphene Fermi level (left). After depositing GQD on the graphene/PZT substrate, the electrons in GQD were transferred to SLG for creating a built-in electric field, following the upward band bending (middle). When under illumination, the photo-induced holes transferred to SLG and reduced the Fermi level of the graphene/PZT device with the assistance of the builtin electric field (right).

Silver nanowire has also been loaded onto the QDs/GR composite to improve optoelectronic performances. In 2016, Liu et al. prepared a CdSe/ZnS QDs/GR/Ag nanowire hybrid by fabricating a rGO-QD-rGO sandwich-structure and loading the $\mathrm{Ag}$ nanowires onto the QD layer. ${ }^{93}$ Compared to the pristine rGO-QD hybrid, this composite demonstrated a 3.6 times enhancement of photocurrent density from 22.1 to $80.3 \mu \mathrm{A}$ $\mathrm{cm}^{-2}$. The enhancement of the optoelectronic conversion efficiency mainly results from the strong quenching ability of silver and the rapid electron transfer of $\mathrm{Ag}$ nanowire and graphene.

Numerous techniques have been applied to improve the responsivity of photodetectors. The QDs/GR composites demonstrate great potential, by taking advantage of the high light harvesting of QDs and high carrier mobility of graphene, and effectively enhancing the sensitivity and gain. However, the defect trap states at the interface of the QDs and graphene limit further development at low illumination power. Besides, under higher illumination power, the responsivity of the photodetector abruptly decreases, due to the screening of the built-in electric field resulting from the accumulation of charges in QDs. To solve this problem, a wise strategy is to apply the PZT substrate in this photodetector. ${ }^{92}$ The intrinsic electric field in PZT effectively contributes to the separation of the photogenerated electron-hole pairs in GQDs and promotes the holes unidirectional transfer to the graphene sheet, resulting in a high responsivity at more than $10^{9} \mathrm{~A} \mathrm{~W}^{-1}$.

As typical photodetectors, phototransistors have much higher gain, responsivity, and signal-to-noise ratio, compared to other types of photodetectors. They have signal amplification ability and can easily be integrated into optoelectronic circuits, data storage circuits, amplifying circuits, rectifier circuits, logical circuits, etc. Phototransistors have been widely applied in detection, automation, optical communication, infrared remote sensing and missile guidance. QDs/GR composites provide a novel route to the manufacture of high quality phototransistors.

Konstantatos et al. reported an extremely high responsivity $\left(\sim 10^{7} \mathrm{~A} \mathrm{~W}^{-1}\right)$ phototransistor based on a $\mathrm{PbS}$ QDs/GR composite fabricated via a spin casting combined layer-bylayer approach. ${ }^{63}$ The schematic of the device and spectral responsivity is shown in Fig. 13. Under illumination, photogenerated electron-hole pairs produced in the PbS QDs, and holes preferred to transfer to graphene; while the electrons remained in the QDs, numerous holes circulated in the channel in graphene, resulting in capacitive coupling and prolonging the carriers' lifetime, and high photoconductive gain was achieved at around $10^{8}$. The small QDs in the PbS QDs/GR composite possessed higher responsivity than the large sized 
(a)

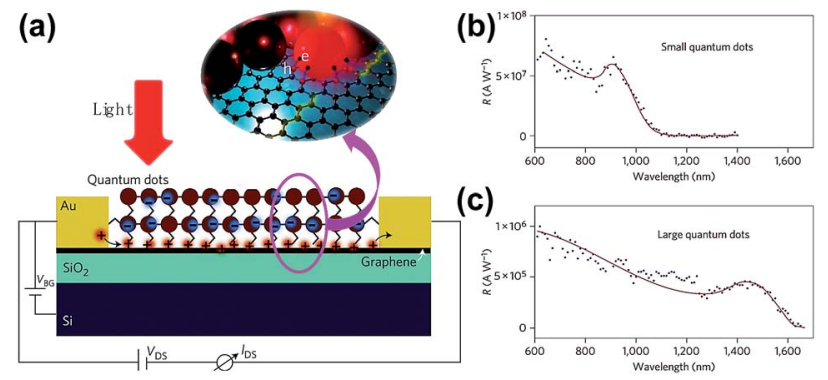

Fig. 13 (a) Schematic illustration of a phototransistor based on the PbS QDs/GR composite. Inset: photo-generated electrons and holes prefer to be transferred to graphene. Spectral responsivity of two devices based on small PbS QDs (b) and large PbS QDs (c). Reprinted with permission from ref. 63. Copyright 2012 Macmillan Publishers Limited.

QDs produced as shown in Fig. 13(b) and (c). The effective electronic coupling of graphene with light-absorbing QDs provides a novel pathway in the applications of optoelectronic devices. Recently, Turyanska et al. ${ }^{59}$ produced a transistor with higher photoresponsivity of $\sim 10^{9} \mathrm{~A} \mathrm{~W}^{-1}$, in which the capped ligands were added to $\mathrm{PbS}$ QDs by drop-casting onto the graphene surface. The capped ligands provided a uniform separation of QDs from graphene and maintained its integrity, which served as a dielectric barrier. The accumulation of charge as well as the short transit time of carriers in graphene contributed to the enhancement of photoresponse and photoconductive gain. As a result, the photoresponsivity of the $\mathrm{PbS}$ QDs/GR composite can be effectively improved by selecting proper ligands.

In 2017, Gong et al. fabricated ZnO QDs/GR heterojunction photodetectors by printing QDs directly on a graphene fieldeffect transistor channel. ${ }^{94}$ This transistor exhibited a high photoresponsivity of $9.9 \times 10^{8} \mathrm{~A} \mathrm{~W}^{-1}$ with a photoconductive gain of $3.6 \times 10^{9}$ for UV light detection. The figure-of-merit UV detectivity attained $10^{14}$ Jones, which was 1 order of magnitude improvement, compared to the best reported nanoscale $\mathrm{ZnO}-$ based UV detectors. The clean van der Waals interface mainly takes responsibility for the facilitation of charge transfer from QDs to graphene. The printing quantum devices combined with graphene demonstrated high performance and low cost for its broad prospect in industrialized application.

\subsection{Lithium ion batteries}

Lithium ion batteries (LIBs), with the advantages of low cost, environmental friendliness and high efficiency, have become one of the most promising energy storage supporters; however, the key factors of high capacity and fast-charging remain to be solved. For the last few decades, the LIBs with anode material based on QDs/GR composites have exhibited excellent performance, which gives this composite significant potential for the LIBs technique. The metal oxide QDs, such as $\mathrm{ZnO},{ }^{28} \mathrm{Fe}_{3} \mathrm{O}_{4}{ }^{29}$ $\mathrm{SnO}_{2},{ }^{47} \mathrm{CoO}^{69}$ and $\mathrm{V}_{2} \mathrm{O}_{5}$ QDs, ${ }^{95}$ decorated with graphene or its derivatives have effectively improved the performance of the LIBs.
Peng et al. used an ultrasonic method to prepare a CoO QD/ GR composite as a LIB anode material. ${ }^{69}$ The reversible lithium storage capacity reached $1592 \mathrm{~mA} \mathrm{~h} \mathrm{~g}^{-1}$ after 50 cycles. The excellent high rate capability and outstanding cycling stability are caused by the large amount of accessible active sites and short diffusion length provided by the CoO QDs. In addition, the $\mathrm{Co}_{3} \mathrm{O}_{4}$ QDs/GR composite also achieved a high reversible capacity of $485 \mathrm{~mA} \mathrm{~h}{ }^{-1}$ for the rate of $5 \mathrm{C}^{.86} \mathrm{Zno}-\mathrm{QDs}$ composites are another common metal oxide material used in LIBs. The ALD-grown ZnO QDs/graphene composite achieved a high capacity of $960 \mathrm{~mA} \mathrm{~h} \mathrm{~g}{ }^{-1}$ under current density of $100 \mathrm{~mA} \mathrm{~g}^{-1} .^{28}$ They demonstrated that the electrochemical performance of electrodes can be improved by controlling the particle size and microstructure. The LIB cycle stability can be maintained while the $\mathrm{ZnO}$ QD sizes are below $7 \mathrm{~nm}$, and smaller QDs exhibit better rate performance. In 2016, Zhao et al. synthesized the $\mathrm{SnO}_{2}$ QDs@GO composite as the anode material in LIBs, which achieved $1121 \mathrm{~mA} \mathrm{~h} \mathrm{~g}^{-1}$ capacity at $100 \mathrm{~mA} \mathrm{~g}^{-1}$; the volume expansion/contraction was effectively released due to this novel core-shell structure and the graphene reticular structure. ${ }^{47}$ The good performance mainly resulted from the conductive substrate and stable solid electrolyte interface (SEI) layer. Yao et al. prepared a $\mathrm{CoFe}_{2} \mathrm{O}_{4} \mathrm{QDs} / \mathrm{N}$-doped GR composite as a LIB anode material by using hydrothermal and calcination methods. ${ }^{96}$ The $\mathrm{CoFe}_{2} \mathrm{O}_{4}$ QDs were 4-12 nm without apparent aggregation on the graphene surface. The manufactured LIBs presented a high initial discharge capacity of $1616 \mathrm{~mA} \mathrm{~h} \mathrm{~g}^{-1}$ and reversible capacity of $1223 \mathrm{~mA} \mathrm{~h} \mathrm{~g}^{-1}$ after 90 cycles at a current of $100 \mathrm{~mA} \mathrm{~g}^{-1}$. Those excellent electrochemical properties can be ascribed to the quantum and size effects of $\mathrm{CoFe}_{2} \mathrm{O}_{4}$ QDs and the synergetic effect of N-doped graphene.

In particular, the modification of rGO by sulfate groups is an admirable strategy for the reduction reaction, which can avoid the use of toxic agents and thermal treatments. Besides, the sulfate groups can serve as a strong bridge to link nanoparticles and graphene, and promote electron transfer. In 2016, Backert

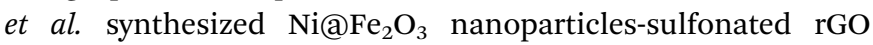
composites as the anode material of LIBs. ${ }^{97}$ The reversible capacity reached to $1051 \mathrm{~mA} \mathrm{~h} \mathrm{~g}{ }^{-1}$ after 40 cycles, which was more than two times higher than that of unwrapped $\mathrm{Ni} @ \mathrm{Fe}_{2} \mathrm{O}_{3}$ (460 $\mathrm{mA} \mathrm{h} \mathrm{g}^{-1}$ ).

Due to the small sizes and quantum effects of QDs, QDs/GR composites can be prepared below critical particle size, ${ }^{98}$ preventing structural collapse during cyclic charge/discharge processes. The reticular structural graphene can buffer its volume expansion/constriction induced in cyclic processes, the excellent graphene conductivity also contributes to quick charge. QDs/GR composites can therefore effectively maintain structural integrity of the active substance, resulting in improving the LIB cyclic stability.

\subsection{Solar cells}

Solar cells are common photovoltaic devices for producing alternative energy. Numerous research works focus on the conversion efficiency improvement. Fortunately, the QDs/GR 
composites provide a novel pathway to promote problem solving.

In 2014, Parand et al. fabricated a GR/PbS composite as the counter electrode for CdS/CdSe QDs sensitized solar cells (QDSC). ${ }^{85}$ The maximum conversion efficiency was $2.63 \%$, which was much higher than that of the sole $\mathrm{PbS}(1.28 \%)$ or graphene $(0.23 \%)$ counter electrode. It is suspected that the GR/ $\mathrm{PbS}$ electrode provides low charge-transfer resistance in order to enhance its conversion efficiency. In 2016, Kim et al. reported the PbS QDs/GR hybrid, as a sandwich structure including 10 layers of tetrabutylammonium iodide (TBAI)-PbS cQDs incorporated with 9 layers graphene sheets and 2 layers of 1,2-ethanedithiol (EDT)-PbS cQDs deposited on ZnO/ITO/glass substrates, achieved a high conversion efficiency of $9.18 \% .^{99}$ The TBAI and EDT solutions served as the exchange ligand and electron blocking/hole transport layer in solar cells. The schematic diagrams of the device, energy level and electron transfer are shown in Fig. 14(a)-(c), respectively. As shown in Fig. 14(d), the $\mathrm{PbS}$ cQDs incorporated with graphene presented an improved photovoltaic performance of $8.82 \pm 0.55 \%$. The PL quenching behavior demonstrated that these materials and sandwich structures bring a fast charge extraction from QDs to graphene flakes and the efficiency is enhanced by adding conductive graphene. Eck et al. fabricated bulk heterojunction solar cells based on a CdSe QDs/GR composite. ${ }^{30}$ The conversion efficiency reached to $4.2 \%$, and the open-circuit voltage was increased by $25-30 \%$ with values up to $0.8 \mathrm{~V}$, compared to the QD only based devices. They also confirmed a higher electron mobility and reduction of trapped charges in the solar cell active layer. A rGO/CuInS ${ }_{2}$ QD hybrid that served as an electron acceptor for polymer-based solar cells was also reported by Meng et al. in $2015 .^{54}$

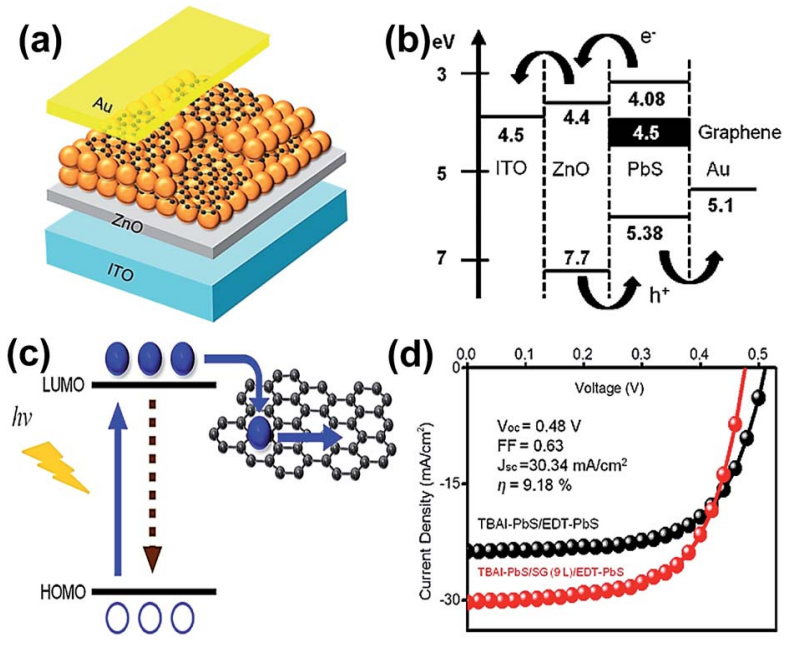

Fig. 14 (a) Schematic diagram of a solar cell based on a colloidal PbS QDs/GR composite. (b) Energy level diagram of the as-obtained device. (c) Emission of electrons and recombination in QDs (left), and their transfer to the graphene sheet (right). (d) $J-V$ characteristics of the solar cell based on TBAI-PbS/EDT-PbS (black dotted line) and TBAI-PbS/GR (9 layers)/EDT-PbS (red dotted line) under illumination of sunlight. Reprinted with permission from ref. 99. Copyright 2016 American Chemical Society.
Due to the properties of low cost, high energy conversion, and simple fabrication procedure, dye-sensitized solar cells (DSSCs) have attracted much attention. In 2016, Chang et al. synthesized GQDs@GR foam nanosheets for application of DSSC electrodes. ${ }^{\mathbf{1 0 0}}$ The fabricated electrodes had a high energy conversion efficiency of $9.59 \%$ with an open-circuit voltage of $0.75 \mathrm{~V}$. The abundant defect sites at the edges of GQDs provided excellent electrocatalytic activity for the reduction of $\mathrm{I}_{3}{ }^{-}$. The high electronic conductivity graphene foam nanosheets act as fast electron transportation channels for promoting the charge transfer from GQDs to the fluorinated tin oxide (FTO) substrate.

Thiol-functionalized rGO attached to QDs also could be applied to active materials in solar cells. In 2016, Madsuha et al. prepared thiol decorated rGO-CdSe QDs and multi-walled carbon nanotubes-CdSe QDs hybrids. ${ }^{\mathbf{1 0 1}}$ The fabricated solar cells achieved initial power conversion efficiency values of about $2 \%$ under AM1.5G illumination. Due to the decoration with thiol, the binding between the QDs and nanocarbon became stronger, which was beneficial for the preservation of the hybrid nanomorphology.

\subsection{Photocatalysis}

In the past few decades, photocatalysis has been the subject of extensive research for applications in environmental pollutant removal and solar energy conversion. Semiconductors are usually used as the light-absorption material, and graphene is an excellent charge transfer material for the enhancement of photocatalysis performance.

In 2015, Gao et al. used a solvothermal method to prepare a $\mathrm{MoS}_{2}$ QD-GR-TiO 2 composite, ${ }^{53}$ and the photocatalytic performance was demonstrated to be 4 times higher than P25. It was found that the $\mathrm{MoS}_{2}$ QDs served as a co-catalyst, which remarkably increased the visible-light absorbance, charge separation efficiency and enhancement of reaction sites. Meanwhile, a report by Tayyebi et al. indicated that a ZnO QDs/ GR composite can enhance the absorption from UV to visible spectra. ${ }^{33}$ The introduced graphene promoted the absorption intensity and restrained the photocorrosion effect. In 2015, a ZnO@PbS QDs/GO composite applied in suspended photocatalytic $\mathrm{H}_{2}$ evolution was reported for the first time. ${ }^{\mathbf{1 0 2}}$ The process of multiple exciton generation (MEG) enhanced the photocatalytic efficiency of hydrogen generation and the separation of electron-hole pairs. It provided a new pathway for QDs/GR composite synthesis with MEG properties. In addition, compared to the pure CdS QDs and graphene sheets, a CdS QDs/GR hybrid, synthesized by the LbL-grown technique, was found to have high photocatalytic activity via UV-visible light spectrometer measurement. ${ }^{32}$

As is well known, $\mathrm{TiO}_{2}$ is widely used as an industrial photocatalyst, due to its properties of good stability, strong oxidation resistance and low toxicity, but the photo-generated electrons and holes recombine quickly because of its wide band gap. It is therefore a challenge to improve $\mathrm{TiO}_{2}$ performance in photocatalysis application. The GQDs/GO composite combined with $\mathrm{TiO}_{2}$ nanotubes provide a new route to solve this problem. Very recently, Tian et al. prepared N, S co-doped GQDs-rGO- 
$\mathrm{TiO}_{2}$ nanotube composites by alkaline hydrothermal and physical stirring methods. ${ }^{103}$ These composites exhibited high photodegradation efficiency that was 1.8 times and 16.3 times higher than those of $\mathrm{rGO}^{-} \mathrm{TiO}_{2}$ nanotube composites and pure $\mathrm{TiO}_{2}$ nanotubes, respectively, for the degradation of methyl orange under visible light irradiation $(\lambda>400 \mathrm{~nm})$. The excellent photocatalytic ability was ascribed to the novel combination of upconversion GQDs and large surface area $\mathrm{rGO}$ and $\mathrm{TiO}_{2}$ nanotubes. The N, S co-doped GQDs can effectively suppress electron-hole recombination and extend the range of visible light, due to its upconversion properties, and the large surface area $\mathrm{rGO}$ and $\mathrm{TiO}_{2}$ nanotubes can improve charge separation and stabilization. Yang et al. reported the ALD-grown $\mathrm{TiO}_{2} \mathrm{QDs} /$ GR composite, which also demonstrated significant photocatalytic enhancement, compared to $\mathrm{P} 25 \mathrm{TiO}_{2}$ and $\mathrm{TiO}_{2}$ film/ GR. ${ }^{104}$

Halide perovskite QDs have also been developed in photocatalytic applications. In 2017, for the first time, Xu et al. synthesized a perovskite composite $\mathrm{CsPbBr}_{3}$ QDs/GO as a photocatalyst to convert $\mathrm{CO}_{2}$ into solar fuel in nonaqueous media. ${ }^{105}$ The rate of electron consumption increased by $25.5 \%$, compared to pure $\mathrm{CsPbBr}_{3}$ QDs; due to the GO improved electron extraction and transport, the PL quenching of $\mathrm{CsPbBr}_{3}$ QDs/GO composite demonstrated the electron transfer from QDs to GO. Meanwhile, the $\mathrm{CsPbBr}_{3}$ QDs/GO composite decayed faster than pure $\mathrm{CsPbBr}_{3}$ QDs with the measured average PL decay time decreasing from 38.3 to $11.3 \mathrm{~ns}$.

\subsection{Electrochemiluminescence sensors}

Owing to the excellent properties of low cost, label-free, and high sensitivity, ECL detection has stimulated significant interest in the preparation of new kinds of materials and exploring the applications. ${ }^{106-108}$ ECL sensors based on QDs/GR composites have been applied in biomedical detection, the detection of cytochrome $\mathrm{C},{ }^{109}$ human IgG, ${ }^{110}$ alpha fetoprotein, ${ }^{111}$ carbohydrate antigen, ${ }^{112}$ and chlorinated phenols. ${ }^{34,113,114}$

Normally, the QDs possess poor biocompatibility and low ECL signals, and have limited applications in bioanalyses. The QDs/GR composites provide a novel pathway for this technique by improving the biocompatibility and ECL signals. Wang et al. fabricated an ECL sensor based on the CdSe QDs/GO-chitosan composite to detect cytochrome C. ${ }^{109}$ The possible ECL mechanisms are as follows:

$$
\begin{gathered}
\mathrm{CdSe}+\mathrm{e}^{-} \rightarrow \mathrm{CdSe}^{\cdot-} \\
\mathrm{S}_{2} \mathrm{O}_{8}{ }^{2-}+\mathrm{e}^{-} \rightarrow \mathrm{SO}_{4}{ }^{2-}+\mathrm{SO}_{4}{ }^{--} \\
\mathrm{CdSe}^{--}+\mathrm{SO}_{4}{ }^{--} \rightarrow \mathrm{CdSe}^{*}+\mathrm{SO}_{4}{ }^{2-} \\
\mathrm{CdSe}^{*} \rightarrow \mathrm{CdSe}+h \nu
\end{gathered}
$$

$\mathrm{K}_{2} \mathrm{~S}_{2} \mathrm{O}_{8}$ served as coreactant; the strong oxidant $\mathrm{SO}_{4}{ }^{-}$radicals and $\mathrm{CdSe}^{--}$anion radicals were produced by the electrochemical reduction of $\mathrm{S}_{2} \mathrm{O}_{8}{ }^{2-}$ and CdSe, respectively. The $\mathrm{SO}_{4}{ }^{--}$ radicals reacted with $\mathrm{CdSe}^{--}$and produced an excited state
(CdSe*), this ECL sensor achieved selective ability for cytochrome $\mathrm{C}$ from bovine serum albumin and glucose, the longterm stability and high ECL intensity were also great. The porous structure of GO-chitosan promotes the loading of CdSe QDs and provides a large interface for electrode reactions. Besides, in this composite, the GO plays an important role in the generation of ECL signals and improves the stability of radicals and makes the fabrication of $\mathrm{SO}_{4}{ }^{--}$and $\mathrm{CdSe}^{--}$easier. The excellent conductivity of GO promotes electron transfer from CdSe QDs to the glass carbon disk (GCE) electrode.

In 2013, Yang et al. developed a carbon QDs/GR composite to detect chlorinated phenols. ${ }^{114}$ The ECL signals were derived from the analytes and carbon QDs with $\mathrm{S}_{2} \mathrm{O}_{8}{ }^{2-}$ serving as coreactant. This ECL sensor achieved real-time detection of pentachlorophenol with ultrahigh sensitivity of $1.0 \times 10^{-12} \mathrm{M}$ concentration in a range from $1.0 \times 10^{-12} \mathrm{M}$ to $1.0 \times 10^{-8} \mathrm{M}$. The eco-friendly and recyclable sensing platform showed ideal recovery rates in real water environments.

\subsection{Supercapacitors}

Supercapacitors have attracted extensive research for energy storage. However, further improving their power density, cycling stability, charge and discharge rates, and reducing its maintenance cost still present a challenge. Fortunately, graphene is an excellent conductive layer that could improve the supercapacitor performance by increasing the electrical conductivity.

The large specific surface area of active composite materials has a significant influence on supercapacitor performances. In 2017, Zhang et al. prepared a GO/carbon dots/polypyrrole composite and used it as an electrode active material. ${ }^{115}$ In this ternary structure, the carbon dots were sandwiched between GO and the polypyrrole layer, which effectively promoted the electron transport and reduced the resistance. The large specific surface area of carbon dots also enhanced the interfacial properties and increased the dielectric constant of the composite. As a result, the manufactured supercapacitor exhibited a high capacitance of $576 \mathrm{~F} \mathrm{~g}^{-1}$ at a current density of $0.5 \mathrm{~A} \mathrm{~g}^{-1}$, and achieved an excellent cycle stability after 5000 cycles. Also, with the consideration of enhancing surface area, Jinlong et al. synthesized a $\mathrm{NiMoO}_{4}$ QDs@nanohole rGO composite that showed better specific capacitance $\left(2327.3 \mathrm{~F} \mathrm{~g}^{-1}\right)$ and cycling stability, compared to the $\mathrm{NiMoO}_{4} @$ rGO composite $\left(1801.2 \mathrm{~F} \mathrm{~g}^{-1}\right){ }^{116}$

Asymmetric supercapacitors (ASC) provide a desirable strategy to improve the performance with fast ion transport. In 2015, Xia et al. synthesized a $\mathrm{Fe}_{2} \mathrm{O}_{3}$ QDs/functionalized GR composite as anode material for ASC. ${ }^{117}$ The fabricated ASC exhibited a high energy density of $50.7 \mathrm{~W} \mathrm{~h} \mathrm{~kg}^{-1}$ at a power of $100 \mathrm{~W} \mathrm{~kg}^{-1}$ and excellent cycling stability. The ionic liquid electrolytes effectively enhanced energy density. In 2016, Shen et al. synthesized a $\mathrm{RuO}_{2} @ \mathrm{C} / \mathrm{rGO}$-based ASC with 1-ethyl-3methylimidazolium tetrafluoroborate $\left(\mathrm{EMIM}-\mathrm{BF}_{4}\right)$ ionic liquid electrolyte. ${ }^{118}$ As shown in Fig. 15(a) and (b), the CV and GCD curves present fast charging/discharging capability and good reversibility. The carbon coated $\mathrm{RuO}_{2} / \mathrm{rGO}$-based ASC 
demonstrated higher specific capacitance, compared to the $\mathrm{RuO}_{2} / \mathrm{rGO}$-based ASC (Fig. 15(c)). Meanwhile, the ASC showed excellent cycling stability and coulombic efficiency with a capacitance of $47.3 \mathrm{~F} \mathrm{~g}^{-1}$ under $3.5 \mathrm{~V}$ potential windows. It also exhibited $98.5 \%$ capacitance retention over 100000 cycles and nearly $100 \%$ coulombic efficiency at the current density of $3 \mathrm{~A} \mathrm{~g}^{-1}$ (Fig. 15(d)).

Highly transparent and flexible supercapacitors based on QDs/GR composites have attracted lots of research interest, due to great potential for use in portable devices. In 2013, He et al. prepared a $3 \mathrm{D} \mathrm{GR} / \mathrm{MnO}_{2}$ composite as the active electrode material of a flexible supercapacitor via electrodeposition. ${ }^{119}$ They achieved a maximum specific capacitance of $465 \mathrm{~F} \mathrm{~g}^{-1}$ under the optimized $\mathrm{MnO}_{2}$ content of $0.1 \mathrm{mg} \mathrm{cm}^{-2}$ at a scan rate of $2 \mathrm{mV} \mathrm{s}^{-1}$. These excellent performances could mainly be ascribed to the high conductivity and porous structure of graphene, and good contact between graphene and $\mathrm{MnO}_{2}$. In 2016, Lee et al. prepared a GQDs-interdigitated pattern of a graphene (ipGR-GQDs) composite via the electrophoretic deposition method. ${ }^{120}$ The fabricated supercapacitor exhibited high energy storage $\left(9.09 \mu \mathrm{F} \mathrm{cm}^{-2}\right)$, stable cycle retention (about $100 \%$ for 10000 cycles), high transparency (92.97\% at $550 \mathrm{~nm})$, and short relaxation time $(8.55 \mathrm{~ms})$. The high stability could be maintained even at a severe bending angle of $45^{\circ}$ with 10000 cycles.

\subsection{Other applications}

QDs/GR composites can also be applied in some emerging applications such as biomarkers, ${ }^{87}$ drug delivery, ${ }^{121}$ gas detection, ${ }^{122} \mathrm{Li}-\mathrm{O}_{2}$ batteries, ${ }^{123}$ sodium ion batteries, ${ }^{124}$ fuel cells, ${ }^{125,126}$ electrocatalysts, ${ }^{127}$ environmental pollutant detection, ${ }^{\mathbf{1 2 8 , 1 2 9}}$ photoelectrochemical determination, ${ }^{\mathbf{1 3 0 - 1 3 4}}$ etc. QDs/ GR hybrids and the QDs/GR composite materials are being further developed, but there are still some drawbacks hindering their extensive applications. The photoelectric responsivity of some QDs/GR hybrids is too low for application in devices, and some photodetectors only present high responsivity in specific narrow wavebands. The performance of QDs/GR-based photodetectors greatly depends on the interfacial states and photogenerated screening field, and therefore, the sensitivity and response time are limited. For solar cells, the conversion efficiency of QDs/GR-based solar cells needs further improvement for industrial applications. Additionally, the cost of manufacturing QD/GR devices is too expensive to meet the large-scale industrial applications. We believe that with the development of scientific research, more QDs/GR composites will be found for application in different fields.

\section{Summary and prospects}

QDs/GR composite materials have attracted much attention due to their excellent effects, and various synthetic methods have been investigated for their fabrication. Herein, we reviewed numerous techniques for QDs decoration on graphene surfaces. With the development of the decoration method, QD/GR composites having multilayer structures were synthesized and widely applied in photodevices. Comparing the different techniques, the proper synthetic methods are highly dependent on the application requirements. Firstly, the hydrothermal/ solvothermal method is unsuitable for the synthesis of QD/ GRs composites for photodevices because it is hard to control the QDs size by this method. The spin-coating method has huge potential for industrial application, due to the controllability of the film thickness. IBSD and MBE techniques have contributed significantly to QD/GR composite synthesis because they produce ordered and size-tunable QDs. The electrochemical method has been demonstrated to be an environmentally (a)
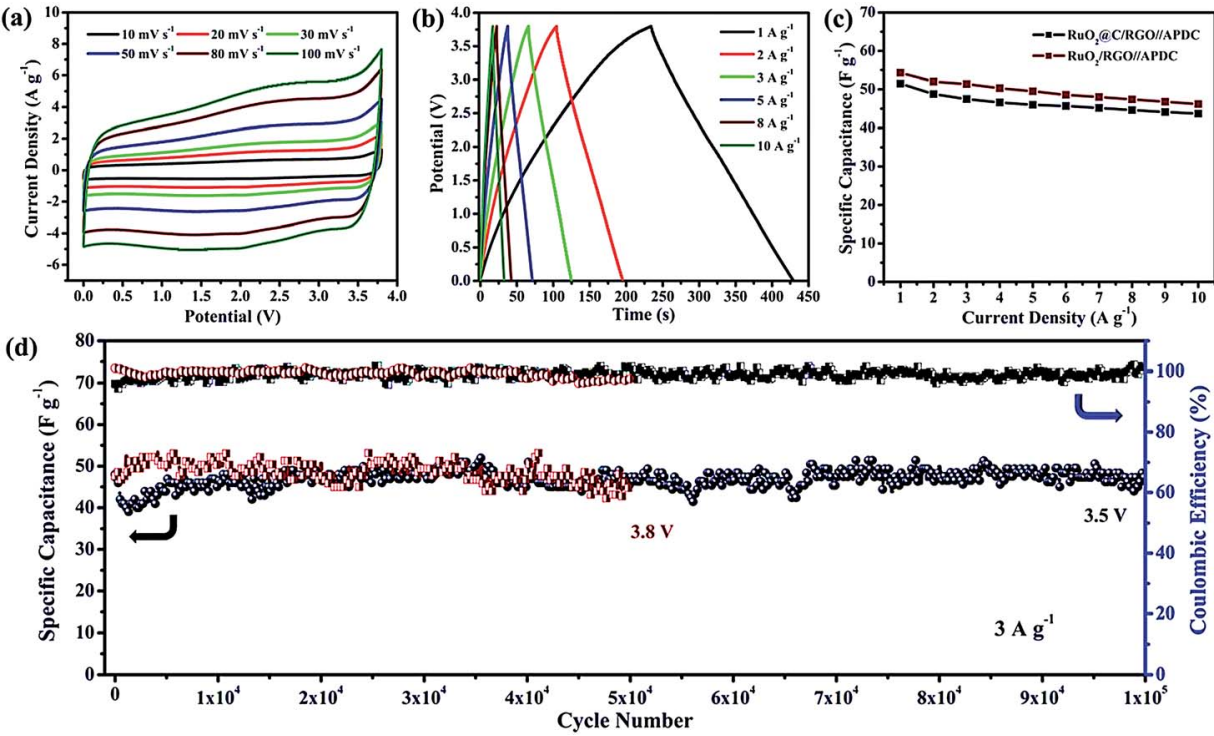

Fig. 15 (a) Cyclic voltammetry (CV) curves and (b) galvanostatic charge/discharge (GCD) curves of the RuO ${ }_{2}$ aC/rGO-based ASC. (c) Specific capacitances of the $\mathrm{RuO}_{2} \mathrm{aC} / \mathrm{rGO}$ and $\mathrm{RuO}_{2} / \mathrm{rGO}$-based ASC. (d) Cycling stability and coulombic efficiency of the RuO ${ }_{2}$ aC/rGO-based ASC. Reprinted with permission from ref. 118. Copyright 2016 Royal Society of Chemistry. 
friendly and low-cost technique, which is adaptable for high performance supercapacitor application.

The insightful strategies for improving the performance of the devices can be summarized as follows. Firstly, the ligands linked or capped with QDs and graphene provide a suitable pathway to fabricate QDs/GR composites and promote their photoelectric properties. The proper choice of species and ligand lengths is essential for performance improvement. The capped ligands can serve as dielectric barriers and maintain the integrity, leading to accumulation and shortening the transit time, resulting in the enhancement of photoresponse and photoconductivity. Secondly, the combined QDs and graphene multilayer sheets have been shown to be excellent structures and are applicable in phototransistors and solar cells. They exhibit the effective transfer of photogenerated electrons to adjacent graphene, prolonging the lifetime of the carriers. The internal series resistance decreases with the increase of graphene layers and is beneficial for the improvement of device performance. Thirdly, the substrate types of the fabricated devices have a significant impact on their performances. For example, the intrinsic electric field of the PZT substrate can improve the separation of photo-hole pairs in QDs and the formed built-in electric field can promote the holes' transfer from the QDs to the graphene channel. There remain various types of substrates for assembly with QDs/GR composites that are worthy of future investigation.

The QDs in the QDs/GR composite mainly provide an outstanding strategy for high-efficiency exciton dissociation, and graphene mainly acts as a highly efficient charge transport channel. This composite has several advantages as follows: (1) strong quantum effects in zero-dimensional QDs, which yield excellent electronic and optoelectronic properties; (2) the high conductivity of graphene benefits the exciton dissociation and charge transfer at the interface with proper interfacial electronic structures; (3) quantum confinement enhances carrier lifetime, which cooperates with graphene's high carrier mobility to improve the responsivity and photoconductive gain.

Preparing ideal QDs/GR composites with high stability, uniform dispersion, tunable size and excellent performance to meet the industrial requirements is however, still a challenge. The preparation of QDs/GR composites by a convenient, lowcost, and established technique is a research breakthrough. We expect to witness the development of QDs/GR composite materials in the next few decades. We believe these novel composites will change the development of science and technology.

\section{Conflicts of interest}

There are no conflicts to declare.

\section{Acknowledgements}

This work was supported by the National Natural Science Foundation of China (No. 11704330, 11564043 and 11504322), the Applied Basic Research Program of Yunnan Province (No. 2016FB006 and 2015FD006), the Scientific Research Key Fund of Yunnan Provincial Department of Education (No. 2015Z002), Yunnan University Action Plan of Serve the Yunnan Province (No. 2016MS15), the Construction Fund of International Joint Research Center for Optoelectronic and Energy Materials by Yunnan Provincial Department of Finance (No. 2017IB033), and the Open Project of National Laboratory for Infrared Physics, Shanghai Institute of Technical Physics, Chinese Academy of Sciences (No. M201509).

\section{References}

1 C. Wang, S. Y. Ke, J. Yang, W. D. Hu, F. Qiu, R. F. Wang and Y. Yang, Nanotechnology, 2015, 26, 105201.

2 C. Fu, H. Wang, T. Song, L. Zhang, W. Li, B. He, M. Sulaman, S. Yang and B. Zou, Nanotechnology, 2016, 27, 065201.

3 K. T. Early and D. J. Nesbitt, Nano Lett., 2015, 15, 7781-7803.

4 M. Tang, S. Chen, J. Wu, Q. Jiang, K. Kennedy, P. Jurczak, M. Liao, R. Beanland, A. Seeds and H. Liu, IEEE J. Sel. Top. Quantum Electron., 2016, 22, 1-7.

5 Y. Li, Y. Wang, C. Chen, A. Pang and M. Wei, Chem, 2012, 18, 11716-11722.

6 W. Yue, M. Lan, G. Zhang, W. Sun, S. Wang and G. Nie, Mater. Sci. Semicond. Process., 2014, 24, 117-125.

7 W. Yue and G. Nie, J. Nanosci. Nanotechnol., 2015, 15, 13121319.

8 K. Bi, W. Z. Gao, T. Q. Zhang, T. Y. Xu, W. W. Zhai and Y. Zhang, Appl. Mech. Mater., 2013, 321-324, 435-438.

9 P. Tománek, S. Changyu, D. Senderáková and M. Hrabovský, Proc. SPIE, 2008, 7138, 71382E.

10 S. Li, Z. H. Ge, B. P. Zhang, Y. Yao, H. C. Wang, J. Yang, Y. Li, C. Gao and Y. H. Lin, Appl. Surf. Sci., 2016, 384, 272-278.

11 S. T. Malak, J. Jung, Y. J. Yoon, M. J. Smith, C. H. Lin, Z. Lin and V. V. Tsukruk, Adv. Opt. Mater., 2016, 4, 608-619.

12 N. Qiao, Z. H. Chen, Y. B. Yang, S. D. Liu, Y. C. Wang and H. Ye, IEEE Photonics J., 2016, 8, 1-7.

13 R. Liang, D. Yan, R. Tian, X. Yu, W. Shi, C. Li, M. Wei, D. G. Evans and X. Duan, Chem. Mater., 2014, 26, 25952600 .

14 D. Deng, K. S. Novoselov, Q. Fu, N. Zheng, Z. Tian and X. Bao, Nat. Nanotechnol., 2016, 11, 218-230.

15 K. S. Novoselov, A. K. Geim and S. V. Morozov, Science, 2004, 306, 666-669.

16 F. Bonaccorso, Z. Sun, T. Hasan and A. C. Ferrari, Nat. Photonics, 2010, 4, 611-622.

17 F. H. Koppens, T. Mueller, P. Avouris, A. C. Ferrari, M. S. Vitiello and M. Polini, Nat. Nanotechnol., 2014, 9, 780-793.

18 F. Cavallo, R. R. Delgado, M. M. Kelly, J. R. S. Pérez, D. P. Schroeder, H. G. Xing, M. A. Eriksson and M. G. Lagally, ACS Nano, 2014, 8, 10237-10245.

19 X. Geng, L. Niu, Z. Xing, R. Song, G. Liu, M. Sun, G. Cheng, H. Zhong, Z. Liu, Z. Zhang, L. Sun, H. Xu, L. Lu and L. Liu, Adv. Mater., 2010, 22, 638-642.

20 C. X. Guo, H. B. Yang, Z. M. Sheng, Z. S. Lu, Q. L. Song and C. M. Li, Angew. Chem., Int. Ed., 2010, 49, 3014-3017. 
21 F. Perreault, A. F. Faria and M. Elimelech, Chem. Soc. Rev., 2015, 44, 5861-5896.

22 Y. Q. Huang, R. J. Zhu, N. Kang, J. Du and H. Q. Xu, Appl. Phys. Lett., 2013, 103, 143119.

23 Y. Gromova, A. Alaferdov, S. Rackauskas, V. Ermakov, A. Orlova, V. Maslov, S. Moshkalev, A. Baranov and A. Fedorov, J. Appl. Phys., 2015, 118, 104305.

24 T. Yu, F. Wang, Y. Xu, L. Ma, X. Pi and D. Yang, Adv. Mater., 2016, 28, 4912-4919.

25 H. Tetsuka, A. Nagoya and S. I. Tamura, Nanoscale, 2016, 8, 19677-19683.

26 W. Guo, S. Xu, Z. Wu, N. Wang, M. M. Loy and S. Du, Small, 2013, 9, 3031-3036.

27 G. Jiang, Y. Su, M. Li, J. Hu, B. Zhao, Z. Yang and H. Wei, RSC Adv., 2016, 6, 97861-97864.

28 X. Sun, C. Zhou, M. Xie, H. Sun, T. Hu, F. Lu, S. M. Scott, S. M. George and J. Lian, J. Mater. Chem. A, 2014, 2, 73197326.

29 S. Zhao, D. Xie, X. Yu, Q. Su, J. Zhang and G. Du, Mater. Lett., 2015, 142, 287-290.

30 M. Eck, C. V. Pham, S. Zufle, M. Neukom, M. Sessler, D. Scheunemann, E. Erdem, S. Weber, H. Borchert, B. Ruhstaller and M. Kruger, Phys. Chem. Chem. Phys., 2014, 16, 12251-12260.

31 M. M. Tavakoli, H. Aashuri, A. Simchi and Z. Fan, Phys. Chem. Chem. Phys., 2015, 17, 24412-24419.

32 F. X. Xiao, J. Miao and B. Liu, J. Am. Chem. Soc., 2014, 136, 1559-1569.

33 A. Tayyebi, M. Outokesh, M. Tayebi, A. Shafikhani and S. S. Şengör, J. Alloys Compd., 2016, 663, 738-749.

34 D. Jiang, X. Du, Q. Liu, L. Zhou, J. Qian and K. Wang, ACS Appl. Mater. Interfaces, 2015, 7, 3093-3100.

35 E. Aktürk, C. Ataca and S. Ciraci, Appl. Phys. Lett., 2010, 96, 123112.

36 J. Y. Hou and S. J. Fonash, Appl. Phys. Lett., 1992, 61, 186188.

37 F. A. Rubinelli, J. Appl. Phys., 1994, 75, 998-1004.

38 C. Main, J. H. Zollondz, S. Reynolds, W. Gao, R. Brüggemann and M. J. Rose, J. Appl. Phys., 1999, 85, 296-301.

39 F. A. Rubinelli, Thin Solid Films, 2016, 619, 102-111.

40 H. Huang, J. Wang, W. Hu, L. Liao, P. Wang, X. Wang, F. Gong, Y. Chen, G. Wu, W. Luo, H. Shen, T. Lin, J. Sun, X. Meng, X. Chen and J. Chu, Nanotechnology, 2016, 27, 445201.

41 M. Khan, M. N. Tahir, S. F. Adil, H. U. Khan, M. R. H. Siddiqui, A. A. Al-warthan and W. Tremel, J. Mater. Chem. A, 2015, 3, 18753-18808.

42 Q. Shi, Y. Cha, Y. Song, J. I. Lee, C. Zhu, X. Li, M. K. Song, D. Du and Y. Lin, Nanoscale, 2016, 8, 15414-15447.

43 C. V. Pham, A. F. Madsuha, T. V. Nguyen and M. Krueger, Synth. Met., 2016, 219, 33-43.

44 E. Ciftci, M. N. Rahaman and M. Shumsky, J. Mater. Sci., 2001, 36, 4875-4882.

45 J. Cao, Q. Liu, J. Du, L. Yang, M. Wei, M. Gao and J. Yang, J. Mater. Sci.: Mater. Electron., 2016, 28, 2267-2271.

46 J. Liu and X. Li, Phys. Lett. A, 2014, 378, 405-407.
47 K. Zhao, L. Zhang, R. Xia, Y. Dong, W. Xu, C. Niu, L. He, M. Yan, L. Qu and L. Mai, Small, 2016, 12, 588-594.

48 P. Ye, H. Chen, L. Bu, K. Zhang and H. Rong, Acta Phys. Sin., 2015, 64, 078102.

49 X. Zhang, F. Xu, B. Zhao, X. Ji, Y. Yao, D. Wu, Z. Gao and K. Jiang, Electrochim. Acta, 2014, 133, 615-622.

50 R. Huang, L. Wang, Q. Zhang, Z. Chen, Z. Li, D. Pan, B. Zhao, M. Wu, C. M. L. Wu and C. H. Shek, ACS Nano, 2015, 9, 11351-11361.

51 H. Fei, R. Ye, G. Ye, Y. Gong, Z. Peng, X. Fan, E. L. G. Samuel, P. M. Ajayan and J. M. Tour, ACS Nano, 2014, 8, 10837-10843.

52 J. He, Y. Chen, P. Li, F. Fu, Z. Wang and W. Zhang, Electrochim. Acta, 2015, 182, 424-429.

53 W. Gao, M. Wang, C. Ran and L. Li, Chem. Commun., 2015, 51, 1709-1712.

54 W. Meng, X. Zhou, Z. Qiu, C. Liu, J. Chen, W. Yue, M. Wang and H. Bi, Carbon, 2016, 96, 532-540.

55 R. Atchudan, T. N. Edison, S. Perumal, D. Karthikeyan and Y. R. Lee, J. Photochem. Photobiol., B, 2016, 162, 500-510.

56 D. Shao, X. Sun, M. Xie, H. Sun, F. Lu, S. M. George, J. Lian and S. Sawyer, Mater. Lett., 2013, 112, 165-168.

57 Z. Zhang, R. F. Wang, J. Zhang, H. S. Li, J. Zhang, F. Qiu, J. Yang, C. Wang and Y. Yang, Nanotechnology, 2016, 27, 305601.

58 D. I. Son, H. Y. Yang, T. W. Kim and W. I. Park, Composites, Part B, 2015, 69, 154-158.

59 L. Turyanska, O. Makarovsky, S. A. Svatek, P. H. Beton, C. J. Mellor, A. Patanè, L. Eaves, N. R. Thomas, M. W. Fay, A. J. Marsden and N. R. Wilson, Adv. Electron. Mater., 2015, 1, 1500062.

60 Y. Gao, O. Roslyak, E. Dervishi, N. S. Karan, Y. Ghosh, C. J. Sheehan, F. Wang, G. Gupta, A. Mohite, A. M. Dattelbaum, S. K. Doorn, J. A. Hollingsworth, A. Piryatinski and H. Htoon, Adv. Opt. Mater., 2015, 3, 3943.

61 D. I. Son, H. Y. Yang, T. W. Kim and W. I. Park, Appl. Phys. Lett., 2013, 102, 021105.

62 X. Liu, X. Ji, M. Liu, N. Liu, Z. Tao, Q. Dai, L. Wei, C. Li, X. Zhang and B. Wang, ACS Appl. Mater. Interfaces, 2015, 7, 2452-2478.

63 G. Konstantatos, M. Badioli, L. Gaudreau, J. Osmond, M. Bernechea, F. P. Garcia de Arquer, F. Gatti and F. H. Koppens, Nat. Nanotechnol., 2012, 7, 363-368.

$64 \mathrm{H}$. Zhang and Y. T. Zhang, MATEC Web Conf., 2016, 44, 01033.

65 Y. Zhang, M. Cao, X. Song, J. Wang, Y. Che, H. Dai, X. Ding, G. Zhang and J. Yao, J. Phys. Chem. C, 2015, 119, 2173921743.

66 Y. Zhang, X. Song, R. Wang, M. Cao, H. Wang, Y. Che, X. Ding and J. Yao, Nanotechnology, 2015, 26, 335201.

67 R. Wang, Y. Zhang, H. Wang, X. Song, L. Jin and J. Yao, IEEE Photonics J., 2015, 7, 4500706.

68 C. Chen, L. Wang, Y. Liu, Z. Chen, D. Pan, Z. Li, Z. Jiao, P. Hu, C. H. Shek, C. M. Wu, J. K. Lai and M. Wu, Langmuir, 2013, 29, 4111-4118. 
69 C. Peng, B. Chen, Y. Qin, S. Yang, C. Li, Y. Zuo, S. Liu and J. Yang, ACS Nano, 2012, 6, 1074-1081.

70 Y. Chen, Q. Wu, Y. Ma, T. Liu, Y. Fan, X. Yang, Z. Zhong, F. Xu, J. Lu and Z. Jiang, Sci. Rep., 2015, 5, 17688.

71 Y. L. Chen, Y. J. Ma, D. D. Chen, W. Q. Wang, K. Ding, Q. Wu, Y. L. Fan, X. J. Yang, Z. Y. Zhong, F. Xu and Z. M. Jiang, Appl. Phys. Lett., 2014, 105, 021104.

72 H. Ajlani, R. Othmen, M. Oueslati, A. Cavanna and A. Madouri, J. Vac. Sci. Technol., B: Nanotechnol. Microelectron.: Mater., Process., Meas., Phenom., 2013, 31, 020602.

73 K. Rezgui, R. Othmen, A. Cavanna, H. Ajlani, A. Madouri and M. Oueslati, J. Raman Spectrosc., 2013, 44, 1529-1533.

74 X. Tan, Y. Li, X. Li, S. Zhou, L. Fan and S. Yang, Chem. Commun., 2015, 51, 2544-2546.

75 L. Tian, S. Yang, Y. Yang, J. Li, Y. Deng, S. Tian, P. He, G. Ding, X. Xie and Z. Wang, $R S C A d v$., 2016, 6, 8264882653.

76 F. Niu, Y. Xu, J. Liu, Z. Song, M. Liu and J. Liu, Electrochim. Acta, 2017, 236, 239-251.

77 J. Deng, Q. Lu, H. Li, Y. Zhang and S. Yao, RSC Adv., 2015, 5, 29704-29707.

78 Y. Li, S. Li, Y. Wang, J. Wang, H. Liu, X. Liu, L. Wang, X. Liu, W. Xue and N. Ma, Phys. Chem. Chem. Phys., 2017, 19, 11631-11638.

79 R. Madhu, B. Dinesh, S. M. Chen, R. Saraswathi and V. Mani, RSC Adv., 2015, 5, 54379-54386.

80 C. Zou, B. Yang, D. Bin, J. Wang, S. Li, P. Yang, C. Wang, Y. Shiraishi and Y. Du, J. Colloid Interface Sci., 2017, 488, 135-141.

81 Y. T. Kim, J. H. Han, B. H. Hong and Y. U. Kwon, Adv. Mater., 2010, 22, 515-518.

82 Q. Chen, Y. Hu, C. Hu, H. Cheng, Z. Zhang, H. Shao and L. Qu, Phys. Chem. Chem. Phys., 2014, 16, 19307-19313.

83 M. Jing, C. Wang, H. Hou, Z. Wu, Y. Zhu, Y. Yang, X. Jia, Y. Zhang and X. Ji, J. Power Sources, 2015, 298, 241-248.

84 H. Yang, C. Guo, G. H. Guai, Q. Song, S. P. Jiang and C. M. Li, ACS Appl. Mater. Interfaces, 2011, 3, 1940-1945.

85 P. Parand, M. Samadpour, A. Esfandiar and A. I. Zad, ACS Photonics, 2014, 1, 323-330.

86 X. Zhou, J. Shi, Y. Liu, Q. Su, J. Zhang and G. Du, Electrochim. Acta, 2014, 143, 175-179.

87 Y. Wang, M. Li, Y. Zhu, S. Ge, J. Yu, M. Yan and X. Song, Analyst, 2013, 138, 7112-7118.

88 C. V. Pham, M. Krueger, M. Eck, S. Weber and E. Erdem, Appl. Phys. Lett., 2014, 104, 132102.

89 C. V. Pham, S. Repp, R. Thomann, M. Krueger, S. Weber and E. Erdem, Nanoscale, 2016, 8, 9682-9687.

90 Z. Sun, Z. Liu, J. Li, G. A. Tai, S. P. Lau and F. Yan, Adv. Mater., 2012, 24, 5878-5883.

91 L. H. Zeng, M. Z. Wang, H. Hu, B. Nie, Y. Q. Yu, C. Y. Wu, L. Wang, J. G. Hu, C. Xie, F. X. Liang and L. B. Luo, ACS Appl. Mater. Interfaces, 2013, 5, 9362-9366.

92 G. Haider, P. Roy, C. W. Chiang, W. C. Tan, Y. R. Liou, H. T. Chang, C. T. Liang, W. H. Shih and Y. F. Chen, Adv. Funct. Mater., 2016, 26, 620-628.
93 B. T. Liu, K. H. Wu and R. H. Lee, Nanoscale Res. Lett., 2016, 11, 388.

94 M. Gong, Q. Liu, B. Cook, B. Kattel, T. Wang, W. L. Chan, D. Ewing, M. Casper, A. Stramel and J. Z. Wu, ACS Nano, 2017, 11, 4114-4123.

95 C. Han, M. Yan, L. Mai, X. Tian, L. Xu, X. Xu, Q. An, Y. Zhao, X. Ma and J. Xie, Nano Energy, 2013, 2, 916-922.

96 L. Yao, H. Deng, Q. A. Huang, Q. Su, X. Du and G. Du, J. Alloys Compd., 2017, 693, 929-935.

97 G. Backert, B. Oschmann, M. N. Tahir, F. Mueller, I. Lieberwirth, B. Balke, W. Tremel, S. Passerini and R. Zentel, J. Colloid Interface Sci., 2016, 478, 155-163.

98 Z. Ma, T. Li, Y. L. Huang, J. Liu, Y. Zhou and D. Xue, RSC $A d v .$, 2013, 3, 7398-7402.

99 B. S. Kim, D. C. Neo, B. Hou, J. B. Park, Y. Cho, N. Zhang, J. Hong, S. Pak, S. Lee, J. I. Sohn, H. E. Assender, A. A. Watt, S. Cha and J. M. Kim, ACS Appl. Mater. Interfaces, 2016, 8, 13902-13908.

100 Q. Chang, Z. Ma, J. Wang, P. Li, Y. Yan, W. Shi, Q. Chen, Y. Huang and L. Huang, Energy Technol., 2016, 4, 256-262.

101 A. F. Madsuha, C. Van Pham, R. Thomann and M. Krueger, Synth. Met., 2016, 222, 34-41.

102 X. F. Shi, X. Y. Xia, G. W. Cui, N. Deng, Y. Q. Zhao, L. H. Zhuo and B. Tang, Appl. Catal., B, 2015, 163, 123-128.

103 H. Tian, K. Shen, X. Hu, L. Qiao and W. Zheng, J. Alloys Compd., 2017, 691, 369-377.

104 L. Yang, L. Jiang, W. Fu, A. W. Weimer, X. Hu and Y. Zhou, Appl. Phys. A, 2017, 123, 416.

105 Y. F. Xu, M. Z. Yang, B. X. Chen, X. D. Wang, H. Y. Chen, D. B. Kuang and C. Y. Su, J. Am. Chem. Soc., 2017, 139, 5660-5663.

106 G. Jie, J. Zhang, D. Wang, C. Cheng, H. Y. Chen and J. J. Zhu, Anal. Chem., 2008, 80, 4033-4039.

107 G. Jie, H. Huang, X. Sun and J. J. Zhu, Biosens. Bioelectron., 2008, 23, 1896-1899.

108 P. Bertoncello and R. J. Forster, Biosens. Bioelectron., 2009, 24, 3191-3200.

109 T. Wang, S. Zhang, C. Mao, J. Song, H. Niu, B. Jin and Y. Tian, Biosens. Bioelectron., 2012, 31, 369-375.

110 L. L. Li, K. P. Liu, G. H. Yang, C. M. Wang, J. R. Zhang and J. J. Zhu, Adv. Funct. Mater., 2011, 21, 869-878.

111 Z. Guo, T. Hao, J. Duan, S. Wang and D. Wei, Talanta, 2012, 89, 27-32.

112 W. Liu, Y. Zhang, S. Ge, X. Song, J. Huang, M. Yan and J. Yu, Anal. Chim. Acta, 2013, 770, 132-139.

113 J. Liang, S. Yang, S. Luo, C. Liu and Y. Tang, Microchim. Acta, 2013, 181, 759-765.

114 S. Yang, J. Liang, S. Luo, C. Liu and Y. Tang, Anal. Chem., 2013, 85, 7720-7725.

115 X. Zhang, J. Wang, J. Liu, J. Wu, H. Chen and H. Bi, Carbon, 2017, 115, 134-146.

116 L. Jinlong, Y. Meng and L. Tongxiang, Appl. Surf. Sci., 2017, 419, 624-630.

117 H. Xia, C. Hong, B. Li, B. Zhao, Z. Lin, M. Zheng, S. V. Savilov and S. M. Aldoshin, Adv. Funct. Mater., 2015, 25, 627-635. 
118 B. Shen, X. Zhang, R. Guo, J. Lang, J. Chen and X. Yan, J. Mater. Chem. A, 2016, 4, 8180-8189.

119 Y. He, W. Chen, X. Li, Z. Zhang, J. Fu, C. Zhao and E. Xie, ACS Nano, 2013, 7, 174-182.

120 K. Lee, H. Lee, Y. Shin, Y. Yoon, D. Kim and H. Lee, Nano Energy, 2016, 26, 746-754.

121 L. Zhang, S. J. Xiao, L. L. Zheng, Y. F. Li and C. Z. Huang, J. Mater. Chem. B, 2014, 2, 8558-8565.

122 Q. Huang, D. Zeng, H. Li and C. Xie, Nanoscale, 2012, 4, 5651-5658.

123 M. N. Samani, H. D. Lim, S. H. Shishavan, H. K. Kim, Y. Ko, M. S. Kim, S. W. Lee, S. F. K. Bozorg, M. Abbasi, H. U. Guim, D. I. Kim, K. C. Roh, K. Kang and K. B. Kim, J. Mater. Chem. A, 2017, 5, 619-631.

124 Y. Wang, Y. Jin, C. Zhao, Y. Duan, X. He and M. Jia, Mater. Lett., 2017, 191, 169-172.

125 M. Fan, C. Zhu, J. Yang and D. Sun, Electrochim. Acta, 2016, 216, 102-109.
126 J. Cao, Y. Hu, L. Chen, J. Xu and Z. Chen, Int. J. Hydrogen Energy, 2017, 42, 2931-2942.

127 M. Govindhan, B. Mao and A. Chen, Nanoscale, 2016, 8, 1485-1492.

128 O. Adegoke, H. Montaseri, S. A. Nsibande and P. B. C. Forbes, J. Alloys Compd., 2017, 720, 70-78.

129 O. Adegoke and P. B. Forbes, Talanta, 2016, 146, 780-788.

130 D. Dutta, S. Chandra, A. K. Swain and D. Bahadur, Anal. Chem., 2014, 86, 5914-5921.

131 Y. Zhu, K. Yan, Y. Liu and J. Zhang, Anal. Chim. Acta, 2015, 884, 29-36.

132 X. Zeng, W. Tu, J. Li, J. Bao and Z. Dai, ACS Appl. Mater. Interfaces, 2014, 6, 16197-16203.

133 R. Wang, K. Yan, F. Wang and J. Zhang, Electrochim. Acta, 2014, 121, 102-108.

134 L. Ruiyi, Q. Sili, L. Zhangyi, L. Ling and L. Zaijun, Sens. Actuators, B, 2017, 250, 372-382. 